\title{
MINIMAL MODEL THEORY FOR LOG SURFACES IN FUJIKI'S CLASS $\mathcal{C}$
}

OSAMU FUJINO

\begin{abstract}
We establish the minimal model theory for $\mathbb{Q}$-factorial log surfaces and $\log$ canonical surfaces in Fujiki's class $\mathcal{C}$.
\end{abstract}

\section{CONTENTS}

1 Introduction 256

2 Preliminaries 259

3 Log surfaces 261

4 Projectivity criteria 263

5 Minimal model program for $\mathbb{Q}$-factorial log surfaces 264

6 Finite generation of log canonical rings 265

7 Abundance theorem 267

8 Contraction theorem for log canonical surfaces 269

9 Log canonical surfaces in Fujiki's class $\mathcal{C}$ with negative Kodaira dimension 271

10 Proof of Theorem 1.5 273

11 Appendix: Vanishing theorems 274

12 Appendix: Complete nonprojective algebraic surfaces 276

References 280

\section{$\S 1$. Introduction}

A $\log$ surface $(X, \Delta)$ in Fujiki's class $\mathcal{C}$ consists of a compact normal analytic surface $X$ that is bimeromorphically equivalent to a compact Kähler manifold and a $\mathbb{Q}$-divisor $\Delta$ on $X$ whose coefficients are in $[0,1] \cap \mathbb{Q}$ such that $K_{X}+\Delta$ is $\mathbb{Q}$-Cartier, that is, there exists a positive integer $m$ such that $m \Delta$ is integral and $\left(\omega_{X}^{\otimes m} \otimes \mathcal{O}_{X}(m \Delta)\right)^{* *}$ is locally free, where $\omega_{X}$ is the canonical sheaf of $X$. In this paper, we establish the following theorem, which is a generalization of the minimal model theory for projective $\mathbb{Q}$-factorial log surfaces obtained in [Fn4] (for some related topics, see [FT], [T], [Ha1], [Li], [Mi], and [Fn7, Section 4.10]).

Theorem 1.1. (Minimal model theory for $\mathbb{Q}$-factorial log surfaces in Fujiki's class $\mathcal{C}$ ) Let $(X, \Delta)$ be a $\mathbb{Q}$-factorial log surface in Fujiki's class $\mathcal{C}$. Then we can construct a finite sequence of projective bimeromorphic morphisms starting from $(X, \Delta)$ :

$$
(X, \Delta)=:\left(X_{0}, \Delta_{0}\right) \stackrel{\varphi_{0}}{\longrightarrow}\left(X_{1}, \Delta_{1}\right) \stackrel{\varphi_{1}}{\longrightarrow} \cdots \stackrel{\varphi_{k-1}}{\longrightarrow}\left(X_{k}, \Delta_{k}\right)=:\left(X^{*}, \Delta^{*}\right)
$$


such that $\left(X_{i}, \Delta_{i}\right)$, where $\Delta_{i}:=\varphi_{i-1 *} \Delta_{i-1}$, is a $\mathbb{Q}$-factorial log surface in Fujiki's class $\mathcal{C}$ and that $\operatorname{Exc}\left(\varphi_{i}\right)=: C_{i} \simeq \mathbb{P}^{1}$ and $-\left(K_{X_{i}}+\Delta_{i}\right) \cdot C_{i}>0$ for every $i$. The final model $\left(X^{*}, \Delta^{*}\right)$ satisfies one of the following conditions.

(i) (Good minimal model). $K_{X^{*}}+\Delta^{*}$ is semiample.

(ii) (Mori fiber space). There exists a surjective morphism $g: X^{*} \rightarrow W$ onto a normal projective variety $W$ with connected fibers such that $-\left(K_{X^{*}}+\Delta^{*}\right)$ is $g$-ample, $\operatorname{dim} W<2$, and the relative Picard number $\rho\left(X^{*} / W\right)$ is one.

We note that

(i) if $X_{i_{0}}$ is projective for some $i_{0}$, then $X_{i}$ is automatically projective for every $i$, and

(ii) if $X_{i_{0}}$ has only rational singularities for some $i_{0}$, then all the singularities of $X_{i}$ are rational for every $i$.

We note that the above sequence of contraction morphisms is nothing but the minimal model program for projective $\mathbb{Q}$-factorial log surfaces established in [Fn4] when $X$ is projective and that $X$ is automatically projective when $\kappa\left(X, K_{X}+\Delta\right)=-\infty$ or 2 .

Theorem 1.1 is not difficult to check once we know the minimal model theory for projective $\mathbb{Q}$-factorial log surfaces in [Fn4], the Enriques-Kodaira classification of compact complex surfaces (see [BHPV, Chapter VI]), and some basic results on complex analytic spaces. We note that Theorem 1.1 includes the abundance theorem for $\mathbb{Q}$-factorial log surfaces in Fujiki's class $\mathcal{C}$.

TheOrem 1.2. (Abundance theorem for $\mathbb{Q}$-factorial log surfaces in Fujiki's class $\mathcal{C}$; see Theorem 7.2) Let $(X, \Delta)$ be a $\mathbb{Q}$-factorial log surface in Fujiki's class $\mathcal{C}$. Assume that $\left(K_{X}+\right.$ $\Delta) \cdot C \geqslant 0$ for every curve $C$ on $X$. Then $K_{X}+\Delta$ is semiample.

From the minimal model theoretic viewpoint, it is very natural to treat log canonical surfaces $(X, \Delta)$ in Fujiki's class $\mathcal{C}$. Unfortunately, $X$ is not necessarily $\mathbb{Q}$-factorial in this case. So we cannot directly apply Theorem 1.1 to $\log$ canonical surfaces in Fujiki's class $\mathcal{C}$. In order to establish the minimal model theory for log canonical surfaces in Fujiki's class $\mathcal{C}$, we prove the following theorem.

Theorem 1.3. (Projectivity of $\log$ canonical surfaces in Fujiki's class $\mathcal{C}$ with negative Kodaira dimension; see Theorem 9.1) Let $(X, \Delta)$ be a log canonical surface in Fujiki's class $\mathcal{C}$. Assume that $\kappa\left(X, K_{X}+\Delta\right)=-\infty$ holds. Then $X$ is projective.

The proof of Theorem 1.3 is much more difficult than we expected. We prove it with the aid of the classification of two-dimensional $\log$ canonical singularities. We note that there are nonprojective normal complete rational surfaces (see [ $\mathrm{Ng}$, Section 4]). Fortunately, such surfaces do not appear under the assumption of Theorem 1.3. Since Nagata's example in $[\mathrm{Ng}$, Section 4] is not log canonical, we explicitly construct some examples of complete nonprojective log canonical algebraic surfaces in Section 12 for the reader's convenience. Our construction, which was suggested by Kento Fujita, is arguably simpler than Nagata's original and classical one (see [Ng, Section 4]). Here, we explain the most interesting example.

EXAMPLE 1.4. (see Example 12.3) There exists a complete nonprojective log canonical algebraic surface $S$ with $\operatorname{Pic}(S)=\{0\}$ and $K_{S} \sim 0$. In particular, $\kappa\left(S, K_{S}\right)=0$ holds. 
For the details of Example 1.4 and some other examples of complete nonprojective algebraic surfaces, see Section 12, where the reader can find some examples of complete nonprojective $\log$ canonical algebraic surfaces $S$ with $\operatorname{Pic}(S)=\{0\}, \overline{\mathrm{NE}}(S)=\mathbb{R}_{\geqslant 0}$, or $\overline{\mathrm{NE}}(S)=N_{1}(S)$.

Thus, by using Theorem 1.3, we have the following minimal model theory for log canonical surfaces in Fujiki's class $\mathcal{C}$.

Theorem 1.5. (Minimal model theory for log canonical surfaces in Fujiki's class $\mathcal{C}$ ) Let $(X, \Delta)$ be a log canonical surface in Fujiki's class $\mathcal{C}$. Then we can construct a finite sequence of projective bimeromorphic morphisms starting from $(X, \Delta)$ :

$$
(X, \Delta)=:\left(X_{0}, \Delta_{0}\right) \stackrel{\varphi_{0}}{\longrightarrow}\left(X_{1}, \Delta_{1}\right) \stackrel{\varphi_{1}}{\longrightarrow} \cdots \stackrel{\varphi_{k-1}}{\longrightarrow}\left(X_{k}, \Delta_{k}\right)=:\left(X^{*}, \Delta^{*}\right)
$$

such that $\left(X_{i}, \Delta_{i}\right)$, where $\Delta_{i}:=\varphi_{i-1} \Delta_{i-1}$, is a log canonical surface in Fujiki's class $\mathcal{C}$ and that $\operatorname{Exc}\left(\varphi_{i}\right)=: C_{i} \simeq \mathbb{P}^{1}$ and $-\left(K_{X_{i}}+\Delta_{i}\right) \cdot C_{i}>0$ for every $i$. The final model $\left(X^{*}, \Delta^{*}\right)$ satisfies one of the following conditions.

(i) (Good minimal model). $K_{X^{*}}+\Delta^{*}$ is semiample.

(ii) (Mori fiber space). There exists a surjective morphism $g: X^{*} \rightarrow W$ onto a normal projective variety $W$ with connected fibers such that $-\left(K_{X^{*}}+\Delta^{*}\right)$ is g-ample, $\operatorname{dim} W<2$, and the relative Picard number $\rho\left(X^{*} / W\right)$ is one.

We note that

(1) if $X_{i_{0}}$ is projective for some $i_{0}$, then $X_{i}$ is automatically projective for every $i$,

(2) if $X_{i_{0}}$ has only rational singularities for some $i_{0}$, then all the singularities of $X_{i}$ are rational for every $i$, and

(3) if $X_{i_{0}}$ is $\mathbb{Q}$-factorial for some $i_{0}$, then so is $X_{i}$ for every $i$.

We note that the above sequence of contraction morphisms is nothing but the usual minimal model program for projective log canonical surfaces (see [Fn 4]) when $X$ is projective and that $X$ is automatically projective when $\kappa\left(X, K_{X}+\Delta\right)=-\infty$ by Theorem 1.3.

In a series of papers (see [HP1], [HP2], and [CHP]), Campana, Höring, and Peternell established the minimal model program and the abundance theorem for Kähler threefolds (see also [HP3]). Their approach is essentially analytic. On the other hand, our approach is much more elementary than theirs and is not analytic. Although we mainly treat compact analytic surfaces in Fujiki's class $\mathcal{C}$, we do not discuss Kähler forms (or currents) on singular surfaces (see $[\mathrm{Fk}]$ ).

In Section 11, we treat some vanishing theorems for proper bimeromorphic morphisms between analytic surfaces. They play an important role in this paper. Although they are more or less known to the experts, we explain the details for the reader's convenience because we can find no suitable references. We think that the results are useful for other applications. The most useful formulation is Theorem 11.3(2).

TheOREM 1.6. (see Theorem 11.3) Let $X$ be a normal analytic surface and let $\Delta$ be an effective $\mathbb{Q}$-divisor on $X$ whose coefficients are less than one such that $K_{X}+\Delta$ is $\mathbb{Q}$-Cartier. Let $f: X \rightarrow Y$ be a proper bimeromorphic morphism onto a normal analytic surface $Y$. Let $\mathcal{L}$ be a line bundle on $X$, and let $D$ be a $\mathbb{Q}$-Cartier Weil divisor on $X$. Assume that $\mathcal{L} \cdot C+$ $\left(D-\left(K_{X}+\Delta\right)\right) \cdot C \geqslant 0$ for every $f$-exceptional curve $C$ on $X$. Then $R^{i} f_{*}\left(\mathcal{L} \otimes \mathcal{O}_{X}(D)\right)=0$ holds for every $i>0$. 
We explain the organization of this paper. In Section 2, we collect some basic definitions and results. In Section 3, we explain a very easy version of the basepoint-free theorem for projective bimeromorphic morphisms between surfaces (see Theorem 3.11). In Section 4, we collect some useful projectivity criteria for $\mathbb{Q}$-factorial compact analytic surfaces. In Section 5, we discuss the minimal model program for $\mathbb{Q}$-factorial log surfaces based on Sakai's contraction theorem, which is a slight generalization of Grauert's famous contraction theorem. Then we prove Theorem 1.1 except for the semiampleness of $K_{X^{*}}+\Delta^{*}$. In Section 6, we briefly discuss the finite generation of log canonical rings of $\mathbb{Q}$-factorial log surfaces, which is essentially contained in [Fn4], and some related topics. In Section 7, we prove the nonvanishing theorem and the abundance theorem. Precisely speaking, we explain how to modify the arguments in $[\mathrm{Fn} 4]$ for $\mathbb{Q}$-factorial log surfaces in Fujiki's class $\mathcal{C}$. In Section 8, we discuss a contraction theorem for log canonical surfaces. A key point is that the exceptional curve automatically becomes $\mathbb{Q}$-Cartier. This simple fact plays a crucial role in our minimal model theory for log canonical surfaces. Section 9 is devoted to the proof of Theorem 1.3, that is, the projectivity of log canonical surfaces in Fujiki's class $\mathcal{C}$ with negative Kodaira dimension. Our proof needs the classification of two-dimensional $\log$ canonical singularities. In Section 10, we prove Theorem 1.5, that is, the minimal model theory for $\log$ canonical surfaces in Fujiki's class $\mathcal{C}$. In Section 11, we discuss some vanishing theorems for proper bimeromorphic morphisms between normal analytic surfaces. Fortunately, we need no deep analytic methods except for the theorem on formal functions for proper morphisms between analytic spaces. In Section 12, we construct some complete nonprojective log canonical algebraic surfaces.

We will use the minimal model theory for projective log surfaces defined over $\mathbb{C}$, the complex number field, established in [Fn4]. We will freely use the basic notation of the minimal model theory as in [Fn3] and [Fn7].

\section{$\S 2 . \quad$ Preliminaries}

In this section, we collect some basic definitions and results.

Definition 2.1. (Boundary and subboundary $\mathbb{Q}$-divisors) Let $X$ be an irreducible normal analytic space and let $\Delta$ be a $\mathbb{Q}$-divisor on $X$. If the coefficients of $\Delta$ are in $[0,1] \cap \mathbb{Q}$ (resp. $(-\infty, 1] \cap \mathbb{Q})$, then $\Delta$ is called a boundary (resp. subboundary) $\mathbb{Q}$-divisor on $X$.

Definition 2.2. (Operations for $\mathbb{Q}$-divisors) Let $D$ be a $\mathbb{Q}$-divisor on a normal analytic space. Then $\lceil D\rceil$ (resp. $\lfloor D\rfloor$ ) denotes the round-up (resp. round-down) of $D$. We put $\{D\}:=$ $D-\lfloor D\rfloor$ and call it the fractional part of $D$.

Definition 2.3. (Algebraic dimensions) Let $X$ be an irreducible compact analytic space. Let $\mathcal{M}(X)$ be the field of meromorphic functions on $X$. Then the transcendence degree of $\mathcal{M}(X)$ over $\mathbb{C}$ is called the algebraic dimension of $X$ and is denoted by $a(X)$. It is well known that $0 \leqslant a(X) \leqslant \operatorname{dim} X$ holds. If $a(X)=\operatorname{dim} X$ holds, then we say that $X$ is Moishezon. We note that if $X$ is Moishezon, then $X$ is an algebraic space, which is proper over $\mathbb{C}$ (see [U, Remark 3.7]).

For the basic properties of $a(X)$, we recommend the reader to see [U, Section 3].

Definition 2.4. Let $X$ be an irreducible compact normal analytic space such that $X$ is Moishezon. Then we can obtain the perfect pairing

$$
N_{1}(X) \times N^{1}(X) \rightarrow \mathbb{R}
$$


induced from the intersection pairing of curves and line bundles as in the case where $X$ is projective. We note that $\rho(X):=\operatorname{dim}_{\mathbb{R}} N^{1}(X)<\infty$ always holds. We call $\rho(X)$ the Picard number of $X$. When $X$ is an algebraic variety, $\overline{\mathrm{NE}}(X)\left(\subset N_{1}(X)\right)$ denotes the Kleiman-Mori cone of $X$.

In this paper, we do not consider $N_{1}(X)$ when $0 \leqslant a(X)<\operatorname{dim} X$. When $X$ is a complete nonprojective singular algebraic variety, $\overline{\mathrm{NE}}(X)$ does not always behave well (see [Fn1], [FP], and Section 12).

REMARK 2.5. Let $X$ be a compact smooth analytic surface whose algebraic dimension $a(X)$ is zero. Then it is well known that there are only finitely many curves on $X$ (see [BHPV, Chapter IV, (8.2) Theorem]).

In the subsequent sections, we will repeatedly use the following well-known negativity lemma and its consequences without mentioning them explicitly. For the proof of Lemma 2.6, see [Ma, Theorem 4-6-1].

LEMma 2.6. (Negativity lemma) Let $P \in Y$ be a germ of normal analytic surface, and let $f: X \rightarrow Y$ be a proper bimeromorphic morphism from a smooth analytic surface $X$. Then $f^{-1}(P)$ is connected and has a negative definite intersection form.

Let us quickly recall the definition of the Iitaka dimension $\kappa$. For the details of $\kappa$, see $[\mathrm{Nk}]$ and $[\mathrm{U}]$.

Definition 2.7. (Iitaka dimensions) Let $X$ be an irreducible compact normal analytic space, and let $\mathcal{L}$ be a line bundle on $X$. Then we set

$$
\kappa(X, \mathcal{L}):=\limsup _{m \rightarrow \infty} \frac{\log \operatorname{dim}_{\mathbb{C}} H^{0}\left(X, \mathcal{L}^{\otimes m}\right)}{\log m}
$$

and call it the Iitaka dimension of $\mathcal{L}$. It is well known that

$$
\kappa(X, \mathcal{L}) \in\{-\infty, 0,1,2, \ldots, \operatorname{dim} X\}
$$

holds. We can define $\kappa(X, D)$ for $\mathbb{Q}$-Cartier $\mathbb{Q}$-divisors $D$ on $X$ similarly.

We close this section with an easy lemma on rational singularities.

Lemma 2.8. (see [Fn6, Lemma 3.1]) Let $\varphi: X \rightarrow Y$ be a proper bimeromorphic morphism between normal analytic spaces. If $R^{i} \varphi_{*} \mathcal{O}_{X}=0$ for every $i>0$, then $X$ has only rational singularities if and only if so does $Y$.

Proof. The problem is local. So we can freely shrink $Y$ around an arbitrary given point. Let us consider a common resolution:

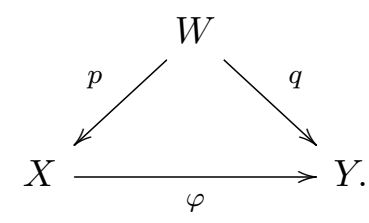

By assumption and the Leray spectral sequence, we have $R^{i} p_{*} \mathcal{O}_{W} \simeq R^{i} q_{*} \mathcal{O}_{W}$ for every $i$. This implies the desired statement. 


\section{$\S 3 . \quad$ Log surfaces}

In this section, we define $\mathbb{Q}$-factorial log surfaces and log canonical surfaces in Fujiki's class $\mathcal{C}$ and discuss a very easy version of the basepoint-free theorem for proper bimeromorphic morphisms between normal analytic surfaces.

In this paper, we adopt the following definition of analytic spaces in Fujiki's class $\mathcal{C}$.

Definition 3.1. (Fujiki's class $\mathcal{C}$ ) Let $X$ be an irreducible compact analytic space. If $X$ is bimeromorphically equivalent to a compact Kähler manifold, then we say that $X$ is in Fujiki's class $\mathcal{C}$.

REMARK 3.2. Let $X$ be an irreducible compact analytic space. We note that if $X$ is Moishezon, then $X$ is automatically in Fujiki's class $\mathcal{C}$.

We have a useful characterization of surfaces in Fujiki's class $\mathcal{C}$.

Lemma 3.3. Let $X$ be an irreducible compact normal analytic surface. Then $X$ is in Fujiki's class $\mathcal{C}$ if and only if there exists a resolution of singularities $f: Y \rightarrow X$ such that $Y$ is Kähler, that is, $Y$ is a two-dimensional compact Kähler manifold and $f$ is a bimeromorphic morphism.

Proof. Note that a compact smooth analytic surface $S$ is Kähler if and only if the first Betti number $b_{1}(S)$ is even (see [BHPV, Chapter IV, (3.1) Theorem]). We also note that the first Betti number is preserved under blow-ups. Thus we can easily check the statement.

As an easy consequence of Lemma 3.3 and its proof, we have the following corollary.

Corollary 3.4. Let $X$ be a compact normal analytic surface in Fujiki's class $\mathcal{C}$. Let $f: Y \rightarrow X$ be any resolution of singularities. Then $Y$ is a compact Kähler manifold.

Let us define canonical sheaves.

Definition 3.5. (Canonical sheaves) Let $X$ be a normal analytic surface and let $\operatorname{Sing} X$ denote the singular locus of $X$. Then we have $\operatorname{codim}_{X} \operatorname{Sing} X \geqslant 2$ since $X$ is normal. Let $\omega_{U}$ be the canonical bundle of $U:=X \backslash \operatorname{Sing} X$. We put $\omega_{X}:=\iota_{*} \omega_{U}$, where $\iota: U \hookrightarrow X$ is the natural open immersion, and call $\omega_{X}$ the canonical sheaf of $X$.

REMARK 3.6. Some normal analytic surface $X$ does not admit any nonzero meromorphic section of $\omega_{X}$. However, if there is no risk of confusion, we use the symbol $K_{X}$ as a formal divisor class with an isomorphism $\mathcal{O}_{X}\left(K_{X}\right) \simeq \omega_{X}$ and call it the canonical divisor of $X$.

In this paper, we adopt the following definition of log surfaces.

DeFinition 3.7. (Log surfaces) Let $X$ be an irreducible compact normal analytic surface, and let $\Delta$ be a boundary $\mathbb{Q}$-divisor on $X$. Assume that $K_{X}+\Delta$ is $\mathbb{Q}$-Cartier. Note that this means that there exists a positive integer $m$ such that $m \Delta$ is integral and that $\left(\omega_{X}^{\otimes m} \otimes \mathcal{O}_{X}(m \Delta)\right)^{* *}$ is locally free. Then the pair $(X, \Delta)$ is called a log surface. We say that a $\log$ surface $(X, \Delta)$ is in Fujiki's class $\mathcal{C}$ when $X$ is in Fujiki's class $\mathcal{C}$. Let $(X, \Delta)$ be a log surface. Then we usually call $\kappa\left(X, K_{X}+\Delta\right)$ the Kodaira dimension of $(X, \Delta)$.

We need to define log canonical surfaces.

Definition 3.8. (Log canonical surfaces) Let $(X, \Delta)$ be a log surface, and let $f: Y \rightarrow$ $X$ be a proper bimeromorphic morphism from a smooth analytic surface $Y$. Then we can 
write $K_{Y}+\Delta_{Y}=f^{*}\left(K_{X}+\Delta\right)$ with $f_{*} \Delta_{Y}=\Delta$. If the coefficients of $\Delta_{Y}$ are less than or equal to one for every $f: Y \rightarrow X$, then $(X, \Delta)$ is called a $\log$ canonical surface.

The notion of $\mathbb{Q}$-factoriality plays a crucial role in this paper.

DeFinition 3.9. (QQQfactoriality) Let $X$ be an irreducible compact normal analytic surface, and let $D$ be a $\mathbb{Q}$-divisor on $X$. Then we say that $D$ is $\mathbb{Q}$-Cartier if there exists a positive integer $m$ such that $m D$ is Cartier. If every Weil divisor on $X$ is $\mathbb{Q}$-Cartier, then we say that $X$ is $\mathbb{Q}$-factorial.

Lemma 3.10 is well known.

Lemma 3.10. Let $X$ be an irreducible compact normal analytic surface. Assume that $X$ has only rational singularities. Then $X$ is $\mathbb{Q}$-factorial.

Proof. This follows from [Nk, Chapter II, 2.12. Lemma].

We close this section with a very easy version of the basepoint-free theorem for projective bimeromorphic morphisms between normal analytic surfaces (see also Remark 11.4).

Theorem 3.11. Let $(X, \Delta)$ be a log surface and let $\varphi: X \rightarrow Y$ be a projective bimeromorphic morphism onto a normal analytic surface $Y$. Assume that $C:=\operatorname{Exc}(\varphi)$ is $\mathbb{Q}$ Cartier, $C \simeq \mathbb{P}^{1}$, and $-C$ and $-\left(K_{X}+\Delta\right)$ are $\varphi$-ample. Let $\mathcal{L}$ be a line bundle on $X$ with $\mathcal{L} \cdot C=0$. Then there exists a line bundle $\mathcal{L}_{Y}$ on $Y$ such that $\mathcal{L} \simeq \varphi^{*} \mathcal{L}_{Y}$ holds. In particular, $X$ is $\mathbb{Q}$-factorial if and only if so is $Y$.

Proof. In Step 1, we will prove the existence of $\mathcal{L}_{Y}$. In Steps 2 and 3, we will see that $X$ is $\mathbb{Q}$-factorial if and only if so is $Y$.

Step 1. Since $C \simeq \mathbb{P}^{1}$ and $\mathcal{L} \cdot C=0,\left.\mathcal{L}\right|_{C}$ is trivial. Since $-C$ and $-\left(K_{X}+\Delta\right)$ are $\varphi$ ample, we may assume that $C \leqslant \Delta$ by increasing the coefficient of $C$ in $\Delta$. Let us consider the following short exact sequence:

$$
0 \rightarrow \mathcal{O}_{X}(-C) \rightarrow \mathcal{O}_{X} \rightarrow \mathcal{O}_{C} \rightarrow 0
$$

Note that

$$
\mathcal{L} \cdot C+\left(-C-\left(K_{X}+\Delta-C\right)\right) \cdot C=\mathcal{L} \cdot C-\left(K_{X}+\Delta\right) \cdot C>0
$$

holds. Therefore, by Theorem 11.3, we get $R^{1} \varphi_{*}\left(\mathcal{L} \otimes \mathcal{O}_{X}(-C)\right)=0$. Thus, we have the following short exact sequence:

$$
0 \rightarrow \varphi_{*}\left(\mathcal{L} \otimes \mathcal{O}_{X}(-C)\right) \rightarrow \varphi_{*} \mathcal{L} \rightarrow \varphi_{*}\left(\left.\mathcal{L}\right|_{C}\right) \rightarrow 0
$$

We note that

$$
\varphi_{*}\left(\left.\mathcal{L}\right|_{C}\right)=H^{0}\left(C,\left.\mathcal{L}\right|_{C}\right) \simeq H^{0}\left(\mathbb{P}^{1}, \mathcal{O}_{\mathbb{P}^{1}}\right) .
$$

By (3.1), $\mathcal{L}$ is $\varphi$-free since $\left.\mathcal{L}\right|_{C}$ is trivial. We note that $\varphi_{*} \mathcal{O}_{X} \simeq \mathcal{O}_{Y}$ since $Y$ is normal and $\varphi$ has connected fibers. Thus $\mathcal{L}_{Y}:=\varphi_{*} \mathcal{L}$ is a line bundle on $Y$ such that $\mathcal{L} \simeq \varphi^{*} \mathcal{L}_{Y}$ holds.

Step 2. Assume that $X$ is $\mathbb{Q}$-factorial. We take a prime divisor $D$ on $Y$. Let $D^{\prime}$ be the strict transform of $D$ on $X$. Then we can take $a \in \mathbb{Q}$ and a divisible positive integer $m$ such that $m\left(D^{\prime}+a C\right)$ is Cartier and $m\left(D^{\prime}+a C\right) \cdot C=0$. We put $\mathcal{L}=\mathcal{O}_{X}\left(m\left(D^{\prime}+a C\right)\right)$ and apply the result obtained in Step 1 to $\mathcal{L}$. Then $m D=\varphi_{*}\left(m\left(D^{\prime}+a C\right)\right)$ is Cartier. This means that $Y$ is $\mathbb{Q}$-factorial. 
Step 3. Assume that $Y$ is $\mathbb{Q}$-factorial. We take a prime divisor $D$ on $X$. Then $D^{\prime}:=\varphi_{*} D$ is a $\mathbb{Q}$-Cartier prime divisor on $Y$. Since $D=\varphi^{*} D^{\prime}-a C$ holds for some $a \in \mathbb{Q}, D$ is $\mathbb{Q}$ Cartier. Therefore, $X$ is $\mathbb{Q}$-factorial.

We complete the proof of Theorem 3.11.

\section{$\S 4$. Projectivity criteria}

Let us start with an easy but very useful projectivity criterion.

Lemma 4.1. (Projectivity of $\mathbb{Q}$-factorial compact analytic surfaces) Let $X$ be a $\mathbb{Q}$ factorial compact analytic surface. Assume that the algebraic dimension $a(X)$ of $X$ is two, that is, $X$ is Moishezon. Then $X$ is projective.

Proof. By the assumption $a(X)=2$, we can construct a proper bimeromorphic morphism $f: Y \rightarrow X$ from a smooth projective surface $Y$. By the assumption $a(X)=2$ again, $X$ is an algebraic space that is proper over $\mathbb{C}$ by Artin's GAGA (see [U, Remark 3.7]). We take a very ample effective Cartier divisor $H$ on $Y$. We put $A=f_{*} H$. Since $X$ is $\mathbb{Q}$-factorial, $A$ is a $\mathbb{Q}$ Cartier divisor. Then we have $A \cdot C=H \cdot f^{*} C>0$ for every curve $C$ on $X$. In particular, we have $A^{2}>0$. Therefore, $A$ is ample by Nakai-Moishezon's ampleness criterion for algebraic spaces (see [P, (1.4) Theorem]). This implies that $X$ is projective.

The following corollary is obvious by Lemma 4.1 .

Corollary 4.2. Let $X$ be a $\mathbb{Q}$-factorial compact analytic surface. Assume that there exists a line bundle $\mathcal{L}$ such that $\kappa(X, \mathcal{L})=2$, that is, $\mathcal{L}$ is a big line bundle. Then $X$ is projective.

Proof. By the assumption that $\mathcal{L}$ is big, we see that the algebraic dimension $a(X)$ of $X$ is two. Therefore, $X$ is projective by Lemma 4.1.

By Corollary 4.2, the minimal model theory for projective $\mathbb{Q}$-factorial log surfaces established in [Fn4] works for $(X, \Delta)$ with $\kappa\left(X, K_{X}+\Delta\right)=2$ in Theorem 1.1.

By combining Lemma 4.1 with the Enriques-Kodaira classification, we obtain the following projectivity criterion.

Lemma 4.3. Let $(X, \Delta)$ be a $\mathbb{Q}$-factorial log surface in Fujiki's class $\mathcal{C}$ with $\kappa\left(X, K_{X}+\right.$ $\Delta)=-\infty$. Then $X$ is projective.

Proof. We take the minimal resolution $f: Y \rightarrow X$. We put $K_{Y}+\Delta_{Y}:=f^{*}\left(K_{X}+\right.$ $\Delta)$. Then we see that $\Delta_{Y}$ is effective by the negativity lemma and that $\kappa\left(Y, K_{Y}+\right.$ $\left.\Delta_{Y}\right)=\kappa\left(X, K_{X}+\Delta\right)=-\infty$ holds. Therefore, we obtain $\kappa\left(Y, K_{Y}\right)=-\infty$ by $\kappa\left(Y, K_{Y}\right) \leqslant$ $\kappa\left(Y, K_{Y}+\Delta_{Y}\right)=-\infty$. Since $X$ is in Fujiki's class $\mathcal{C}$, the first Betti number $b_{1}(Y)$ of $Y$ is even. Therefore, by the Enriques-Kodaira classification (see [BHPV, Chapter VI]), $Y$ is a smooth projective surface. Thus, by Lemma $4.1, X$ is projective.

We will repeatedly use the above projectivity criteria throughout this paper.

We note that the statement of Theorem 1.3 looks very similar to that of Lemma 4.3. However, a log canonical surface is not necessarily $\mathbb{Q}$-factorial. Therefore, Theorem 1.3 is much harder to prove than Lemma 4.3 (see Section 9). 


\section{$\S 5$. Minimal model program for $\mathbb{Q}$-factorial log surfaces}

By repeatedly using Grauert's contraction theorem, we can easily run a kind of the minimal model program for $\mathbb{Q}$-factorial $\log$ surfaces $(X, \Delta)$. We note that $X$ is not assumed to be in Fujiki's class $\mathcal{C}$ in Theorem 5.1. A key point of Theorem 5.1 is the assumption that $X$ is $\mathbb{Q}$-factorial.

Theorem 5.1. Let $(X, \Delta)$ be a compact $\mathbb{Q}$-factorial log surface. We assume that $\kappa\left(X, K_{X}+\Delta\right) \geqslant 0$. Then we can construct a finite sequence of projective bimeromorphic morphisms

$$
(X, \Delta)=:\left(X_{0}, \Delta_{0}\right) \stackrel{\varphi_{0}}{\longrightarrow}\left(X_{1}, \Delta_{1}\right) \stackrel{\varphi_{1}}{\longrightarrow} \cdots \stackrel{\varphi_{k-1}}{\longrightarrow}\left(X_{k}, \Delta_{k}\right)=:\left(X^{*}, \Delta^{*}\right)
$$

with $\Delta_{i}:=\varphi_{i-1 *} \Delta_{i-1}, \operatorname{Exc}\left(\varphi_{i}\right)=: C_{i} \simeq \mathbb{P}^{1}$, and $-\left(K_{X_{i}}+\Delta_{i}\right) \cdot C_{i}>0$ for every $i$ such that $\left(K_{X^{*}}+\Delta^{*}\right) \cdot C \geqslant 0$ for every curve $C$ on $X^{*}$. We note that $\left(X_{i}, \Delta_{i}\right)$ is a compact $\mathbb{Q}$-factorial log surface for every $i$.

Proof. Since $\kappa\left(X, K_{X}+\Delta\right) \geqslant 0$, we can take an effective Cartier divisor $D \in \mid m\left(K_{X}+\right.$ $\Delta) \mid$ for some large and divisible positive integer $m$. If $m\left(K_{X}+\Delta\right) \cdot C=D \cdot C \geqslant 0$ for every curve $C$ on $X$, then we set $\left(X^{*}, \Delta^{*}\right):=\left(X_{0}, \Delta_{0}\right)=(X, \Delta)$. So we assume that there exists some irreducible curve $C$ on $X$ such that $D \cdot C<0$. Then $C$ is an irreducible component of Supp $D$ and $C^{2}<0$. By Sakai's contraction theorem (see [S, Theorem (1.2)]), which is a slight generalization of Grauert's famous contraction theorem, we get a bimeromorphic morphism $\varphi_{0}: X=X_{0} \rightarrow X_{1}$ that contracts $C$ to a normal point of $X_{1}$. We take a divisible positive integer $l$ such that $l C$ is Cartier. Then $\mathcal{O}_{X}(-l C)$ is a $\varphi_{0}$-ample line bundle on $X$. In particular, $\varphi_{0}$ is a projective morphism. By construction, $-\left(K_{X}+\Delta\right) \cdot C>0$. Therefore, $-\left(K_{X}+\Delta\right)$ is $\varphi_{0}$-ample. Thus, $R^{i} \varphi_{0 *} \mathcal{O}_{X}=0$ for every $i>0$ by Theorem 11.3.

Claim 1. $C$ is isomorphic to $\mathbb{P}^{1}$.

Proof of Claim 1. We consider the following exact sequence:

$$
\cdots \rightarrow R^{1} \varphi_{0 *} \mathcal{O}_{X} \rightarrow R^{1} \varphi_{0 *} \mathcal{O}_{C} \rightarrow R^{2} \varphi_{0 *} \mathcal{I}_{C} \rightarrow \cdots
$$

where $\mathcal{I}_{C}$ is the defining ideal sheaf of $C$ on $X$. As we saw above, $R^{1} \varphi_{0 *} \mathcal{O}_{X}=0$ holds. Since $C$ is a curve, $R^{2} \varphi_{0 *} \mathcal{I}_{C}=0$ holds by the theorem on formal functions for proper morphisms between analytic spaces (see [BS, Chapter VI, Corollary 4.7]). Thus we get $H^{1}\left(C, \mathcal{O}_{C}\right)=R^{1} \varphi_{0 *} \mathcal{O}_{C}=0$ by the above exact sequence. This implies that $C$ is isomorphic to $\mathbb{P}^{1}$.

Therefore, by Theorem 3.11, we obtain that $\left(X_{1}, \Delta_{1}\right)$ is a $\mathbb{Q}$-factorial log surface. Since Supp $D$ has only finitely many irreducible components, we get a desired sequence of contraction morphisms and finally obtain $\left(X^{*}, \Delta^{*}\right)$ with $\left(K_{X^{*}}+\Delta^{*}\right) \cdot C \geqslant 0$ for every curve $C$ on $X^{*}$.

We note the following well-known lemma on extremal rays of projective surfaces.

Lemma 5.2. Let $X$ be a normal projective surface, and let $C$ be a $\mathbb{Q}$-Cartier irreducible curve on $X$ with $C^{2}<0$. Then the numerical equivalence class $[C]$ of $C$ spans an extremal ray of the Kleiman-Mori cone $\overline{\mathrm{NE}}(X)$ of $X$.

Proof. This is obvious. For the proof, see [KM, Lemma 1.22]. 
By Lemma 5.2, if $X$ is projective in Theorem 5.1, then the minimal model program in Theorem 5.1 is nothing but the minimal model program for projective $\mathbb{Q}$-factorial log surfaces formulated and established in [Fn4]. We also note that $X$ is projective in Theorem 5.1 if the algebraic dimension $a(X)$ of $X$ is two by Lemma 4.1.

We recall that $\mathbb{Q}$-factorial $\log \operatorname{surfaces}(X, \Delta)$ in Fujiki's class $\mathcal{C}$ with $\kappa\left(X, K_{X}+\Delta\right)=$ $-\infty$ are projective by Lemma 4.3 .

Let us prove Theorem 1.1 except for the semiampleness of $K_{X^{*}}+\Delta^{*}$.

Proof of Theorem 1.1. If $\left(K_{X}+\Delta\right) \cdot C \geqslant 0$ for every curve $C$ on $X$, then we put $\left(X^{*}, \Delta^{*}\right):=(X, \Delta)$. We will see that $K_{X^{*}}+\Delta^{*}$ is semiample in Theorem 7.2. We note that $X$ is in Fujiki's class $\mathcal{C}$. If $\kappa\left(X, K_{X}+\Delta\right)=-\infty$, then $X$ is projective by Lemma 4.3. Therefore, we can run the minimal model program for $\mathbb{Q}$-factorial projective $\log$ surfaces in [Fn4] and finally get a Mori fiber space. Therefore, we may further assume that $\kappa\left(X, K_{X}+\Delta\right) \geqslant 0$. Then we can apply Theorem 5.1 and finally get a model $\left(X^{*}, \Delta^{*}\right)$ such that $\left(K_{X^{*}}+\Delta^{*}\right) \cdot C \geqslant 0$ for every curve $C$ on $X^{*}$. In this case, by the abundance theorem, Theorem 7.2, we will see that $K_{X^{*}}+\Delta^{*}$ is semiample.

Since we have $R^{1} \varphi_{i_{*}} \mathcal{O}_{X_{i}}=0$ (see Theorem 11.3), $X_{i}$ has only rational singularities if and only if so does $X_{i+1}$ by Lemma 2.8. Thus we have (2).

Since each contraction $\varphi_{i}$ is projective, $X_{i}$ is projective when so is $X_{i+1}$. On the other hand, if $X_{i}$ is projective, then so is $X_{i+1}$ because $\varphi_{i}$ is nothing but the usual contraction morphism associated with a $\left(K_{X_{i}}+\Delta_{i}\right)$-negative extremal ray (see Lemma 5.2). Thus, we have (1).

We obtained Theorem 1.1 except for the semiampleness of $K_{X^{*}}+\Delta^{*}$, which will be proved in Section 7.

\section{$\S 6$. Finite generation of log canonical rings}

In this section, we briefly discuss the finite generation of log canonical rings of pairs for the reader's convenience.

The following theorem is the main result of this section, which is essentially contained in [Fn4].

TheOREM 6.1. (Finite generation of $\log$ canonical rings) Let $(X, \Delta)$ be a compact $\mathbb{Q}$ factorial log surface. Then the log canonical ring

$$
\bigoplus_{m \geqslant 0} H^{0}\left(X, \mathcal{O}_{X}\left(\left\lfloor m\left(K_{X}+\Delta\right)\right\rfloor\right)\right)
$$

is a finitely generated $\mathbb{C}$-algebra. We note that the sheaf $\mathcal{O}_{X}\left(\left\lfloor m\left(K_{X}+\Delta\right)\right\rfloor\right)$ denotes $\left(\omega_{X}^{\otimes m} \otimes\right.$ $\left.\mathcal{O}_{X}(\lfloor m \Delta\rfloor)\right)^{* *}$.

As an easy consequence of Theorem 6.1, we have the following corollary.

Corollary 6.2. Let $(X, \Delta)$ be a compact log canonical surface. Then the log canonical ring

$$
\bigoplus_{m \geqslant 0} H^{0}\left(X, \mathcal{O}_{X}\left(\left\lfloor m\left(K_{X}+\Delta\right)\right\rfloor\right)\right)
$$

is a finitely generated $\mathbb{C}$-algebra.

We note that $X$ is not assumed to be in Fujiki's class $\mathcal{C}$ in Theorem 6.1 and Corollary 6.2. 
Proof of Corollary 6.2. Let $f: Y \rightarrow X$ be the minimal resolution. We put $K_{Y}+\Delta_{Y}:=$ $f^{*}\left(K_{X}+\Delta\right)$. Since $(X, \Delta)$ is $\log$ canonical, we see that $\Delta_{Y}$ is a boundary $\mathbb{Q}$-divisor by the negativity lemma. By Theorem 6.1 , the $\log$ canonical ring of $\left(Y, \Delta_{Y}\right)$ is a finitely generated $\mathbb{C}$-algebra. This implies that the $\log$ canonical ring of $(X, \Delta)$ is a finitely generated $\mathbb{C}$ algebra.

Before we prove Theorem 6.1, let us recall the following easy well-known lemma for the reader's convenience.

Lemma 6.3. Let $X$ be an irreducible compact normal analytic space and let $\mathcal{L}$ be a line bundle on $X$ such that $\kappa(X, \mathcal{L}) \leqslant 1$. Then the graded ring

$$
R(X, \mathcal{L}):=\bigoplus_{m \geqslant 0} H^{0}\left(X, \mathcal{L}^{\otimes m}\right)
$$

is a finitely generated $\mathbb{C}$-algebra.

Sketch of Proof. If $\kappa(X, \mathcal{L})=-\infty$ or 0 , then it is very easy to see that $R(X, \mathcal{L})$ is a finitely generated $\mathbb{C}$-algebra. If $\kappa(X, \mathcal{L})=1$, then we can reduce the problem to the case where $X$ is a smooth projective curve and $\mathcal{L}$ is an ample line bundle on $X$ by taking the Iitaka fibration (see $[\mathrm{Mo},(1.12)$ Theorem]). Thus, $R(X, \mathcal{L})$ is a finitely generated $\mathbb{C}$-algebra when $\kappa(X, \mathcal{L}) \leqslant 1$.

Let us prove Theorem 6.1 .

Proof of Theorem 6.1. By Lemma 6.3, we may assume that $\kappa\left(X, K_{X}+\Delta\right)=2$. Then, by Corollary $4.2, X$ is projective. In this case, the log canonical ring

$$
\bigoplus_{m \geqslant 0} H^{0}\left(X, \mathcal{O}_{X}\left(\left\lfloor m\left(K_{X}+\Delta\right)\right\rfloor\right)\right)
$$

of $(X, \Delta)$ is a finitely generated $\mathbb{C}$-algebra by the minimal model theory for projective $\mathbb{Q}$ factorial log surfaces established in [Fn4].

Let us quickly see some results and conjectures on log canonical rings of higherdimensional pairs.

Theorem 6.4. ([BCHM], [FM], and [Fn5, Theorem 1.8]) Let $(X, \Delta)$ be a Kawamata log terminal pair such that $\Delta$ is a $\mathbb{Q}$-divisor on $X$ and that $X$ is in Fujiki's class $\mathcal{C}$. Then the log canonical ring

$$
\bigoplus_{m \geqslant 0} H^{0}\left(X, \mathcal{O}_{X}\left(\left\lfloor m\left(K_{X}+\Delta\right)\right\rfloor\right)\right)
$$

is a finitely generated $\mathbb{C}$-algebra.

Conjecture 6.5. Let $(X, \Delta)$ be a log canonical pair such that $\Delta$ is a $\mathbb{Q}$-divisor on $X$ and that $X$ is in Fujiki's class $\mathcal{C}$. Then the log canonical ring

$$
\bigoplus_{m \geqslant 0} H^{0}\left(X, \mathcal{O}_{X}\left(\left\lfloor m\left(K_{X}+\Delta\right)\right\rfloor\right)\right)
$$

is a finitely generated $\mathbb{C}$-algebra. 
Conjecture 6.5 is still widely open even when $X$ is projective (see [Fn2], [Fn5], [FG], [Ha2], and [FL]). When $X$ is projective in Conjecture 6.5, it is essentially equivalent to the existence problem of good minimal models for lower-dimensional varieties (for the details, see $[\mathrm{FG}]$ ). Note that Corollary 6.2 completely settled Conjecture 6.5 in dimension two.

We close this section with a naive question.

QuESTION 6.6. Let $X$ be an irreducible compact normal analytic surface such that $K_{X}$ is $\mathbb{Q}$-Cartier. Then, is the canonical ring

$$
\bigoplus_{m \geqslant 0} H^{0}\left(X, \mathcal{O}_{X}\left(m K_{X}\right)\right)
$$

a finitely generated $\mathbb{C}$-algebra?

We do not know the answer even when $X$ is projective.

\section{§7. Abundance theorem}

In this section, we prove the abundance theorem for $\mathbb{Q}$-factorial log surfaces in Fujiki's class $\mathcal{C}$.

Let us start with the nonvanishing theorem.

Theorem 7.1. (Nonvanishing theorem) Let $(X, \Delta)$ be a $\mathbb{Q}$-factorial log surface in Fujiki's class $\mathcal{C}$. Assume that $\left(K_{X}+\Delta\right) \cdot C \geqslant 0$ for every curve $C$ on $X$. Then we have $\kappa\left(X, K_{X}+\Delta\right) \geqslant 0$.

Proof. Let $f: Y \rightarrow X$ be the minimal resolution. We put $K_{Y}+\Delta_{Y}:=f^{*}\left(K_{X}+\Delta\right)$. Then $\Delta_{Y}$ is an effective $\mathbb{Q}$-divisor by the negativity lemma. If $\kappa\left(Y, K_{Y}\right) \geqslant 0$, then we have

$$
\kappa\left(X, K_{X}+\Delta\right)=\kappa\left(Y, K_{Y}+\Delta_{Y}\right) \geqslant \kappa\left(Y, K_{Y}\right) \geqslant 0 .
$$

Therefore, from now on, we assume that $\kappa\left(Y, K_{Y}\right)=-\infty$. By Lemma 4.3, $Y$ is projective. Therefore, by Lemma 4.1, $X$ is projective since $X$ is $\mathbb{Q}$-factorial by assumption. Thus, by [Fn4, Theorem 5.1], we get $\kappa\left(X, K_{X}+\Delta\right) \geqslant 0$.

The following theorem is the main result of this section, which is the abundance theorem for $\mathbb{Q}$-factorial log surfaces in Fujiki's class $\mathcal{C}$.

TheOREm 7.2. (Abundance theorem for $\mathbb{Q}$-factorial log surfaces in Fujiki's class $\mathcal{C}$ ) Let $(X, \Delta)$ be a $\mathbb{Q}$-factorial log surface in Fujiki's class $\mathcal{C}$. Assume that $\left(K_{X}+\Delta\right) \cdot C \geqslant 0$ for every curve $C$ on $X$. Then $K_{X}+\Delta$ is semiample.

For the proof of Theorem 7.2, we prepare some easy lemmas.

Lemma 7.3. Let $X$ be a compact normal analytic surface, and let $\mathcal{L}$ be a line bundle on $X$ such that $\mathcal{L} \cdot C \geqslant 0$ for every curve $C$ on $X$. Assume that $\kappa(X, \mathcal{L})=1$. Then $\mathcal{L}$ is semiample.

Proof. This is an easy consequence of Zariski's lemma (see [BHPV, Chapter III, (8.2) Lemma]). For the details, see [Ft, (4.1) Theorem].

LEMma 7.4. Let $S$ be a compact smooth analytic surface in Fujiki's class $\mathcal{C}$ with $\kappa\left(S, K_{S}\right)=0$. Assume that the algebraic dimension $a(S)$ of $S$ is less than two. Then $S$ is bimeromorphically equivalent to a K3 surface or a two-dimensional complex torus. 
Proof. Since $S$ is in Fujiki's class $\mathcal{C}$, the first Betti number $b_{1}(S)$ of $S$ is even. Then the Enriques-Kodaira classification (see [BHPV, Chapter VI]) and $\kappa\left(S, K_{S}\right)=0$ give the desired statement.

Lemma 7.5. Let $B$ be a nonzero effective divisor on a two-dimensional complex torus $S$. Then we have $\kappa(S, B) \geqslant 1$.

Proof. Without loss of generality, we may assume that $B$ is an irreducible curve on $S$. If $B$ is not an elliptic curve, then we can see that $S$ is an Abelian surface (see [U, Lemma 10.8]). In this case, it is well known that $|2 B|$ is basepoint-free. In particular, $\kappa(S, B) \geqslant 1$. Therefore, from now on, we assume that $B$ is an elliptic curve. By taking a suitable translation, we may further assume that $B$ is a complex subtorus of $S$. We set $A=S / B$. Let $p: S \rightarrow A$ be the canonical quotient map. Then $B=p^{*} P$ holds for $P=p(B) \in A$. Therefore, we obtain $\kappa(S, B)=\kappa(A, P)=1$. Hence, we always have $\kappa(S, B) \geqslant 1$.

Lemma 7.6. Let $S$ be a $K 3$ surface, and let $B$ be a nonzero effective divisor on $S$ such that $B^{2}=0$. Then we have $\kappa(S, B) \geqslant 1$.

Proof. By the Riemann-Roch formula,

$$
\operatorname{dim} H^{0}\left(S, \mathcal{O}_{S}(B)\right)+\operatorname{dim} H^{2}\left(S, \mathcal{O}_{S}(B)\right) \geqslant \chi\left(S, \mathcal{O}_{S}\right)=2
$$

By Serre duality,

$$
H^{2}\left(S, \mathcal{O}_{S}(B)\right) \simeq H^{0}\left(S, \mathcal{O}_{S}(-B)\right) .
$$

Since $B$ is a nonzero effective divisor on $S, H^{0}\left(S, \mathcal{O}_{S}(-B)\right)=0$ and $\operatorname{dim} H^{0}\left(S, \mathcal{O}_{S}(B)\right) \geqslant 2$. Therefore, we have $\kappa(S, B) \geqslant 1$.

Before we prove Theorem 7.2, we explicitly state the abundance theorem for log canonical surfaces in Fujiki's class $\mathcal{C}$.

Corollary 7.7. (Abundance theorem for log canonical surfaces in Fujiki's class $\mathcal{C}$ ) Let $(X, \Delta)$ be a log canonical surface in Fujiki's class $\mathcal{C}$. Assume that $\left(K_{X}+\Delta\right) \cdot C \geqslant 0$ for every curve $C$ on $X$. Then $K_{X}+\Delta$ is semiample.

Proof. Let $f: Y \rightarrow X$ be the minimal resolution of $X$. We put $K_{Y}+\Delta_{Y}:=f^{*}\left(K_{X}+\Delta\right)$. Then $\Delta_{Y}$ is effective by the negativity lemma and is a subboundary $\mathbb{Q}$-divisor on $Y$ since $(X, \Delta)$ is $\log$ canonical. Therefore, $\Delta_{Y}$ is a boundary $\mathbb{Q}$-divisor on $Y$. We can easily see that $\left(K_{Y}+\Delta_{Y}\right) \cdot C_{Y} \geqslant 0$ for every curve $C_{Y}$ on $Y$. Thus, by Theorem $7.2, K_{Y}+\Delta_{Y}$ is semiample. This implies that $K_{X}+\Delta$ is also semiample.

Let us start the proof of Theorem 7.2.

Proof of Theorem 7.2. By the nonvanishing theorem (see Theorem 7.1), we have $\kappa\left(X, K_{X}+\Delta\right) \geqslant 0$.

Step 1. $(\kappa=2)$ If $\kappa\left(X, K_{X}+\Delta\right)=2$, then $X$ is projective by Corollary 4.2. In this case, we can apply [Fn4, Theorem 4.1], which is one of the deepest results in [Fn4], and obtain that $K_{X}+\Delta$ is semiample.

Step 2. $(\kappa=1)$ If $\kappa\left(X, K_{X}+\Delta\right)=1$, then we see that $K_{X}+\Delta$ is semiample by Lemma 7.3. 
Step 3. $(\kappa=0)$ In this step, we assume $\kappa\left(X, K_{X}+\Delta\right)=0$. If $X$ is projective, then $K_{X}+\Delta$ is semiample by [Fn4, Theorem 6.2]. Here, we will explain that the proof of [Fn4, Theorem 6.2] works with some minor modifications when $X$ is not projective. From now on, we will freely use the notation of the proof of [Fn4, Theorem 6.2].

The first part of the proof of [Fn4, Theorem 6.2] works without any changes (see page 361 in [Fn4]). We note that $Z$ is a member of $\left|m\left(K_{S}+\Delta_{S}\right)\right|$ for some divisible positive integer $m$. We also note that Mumford's arguments on indecomposable curves of canonical type work on smooth analytic surfaces (see [Mu2, Definition, Lemma, and Corollary 1 in Section 2]). Therefore, [Fn4, Lemma 6.3] holds true. In particular, we obtain that $Z^{2}=0$. The compact smooth surface $S$ constructed in the first part of [Fn4, Theorem 6.2] is not projective. Of course, $S$ is in Fujiki's class $\mathcal{C}$ because $S$ is bimeromorphically equivalent to $X$ by construction. As in the proof of [Fn4, Theorem 6.2], we will derive a contradiction assuming $Z \neq 0$.

By Lemma 4.3 , we have $\kappa\left(S, K_{S}\right) \geqslant 0$ since $S$ is not projective. Thus, all we have to do is to check that Step 1 in the proof of [Fn4, Theorem 6.2] works when $S$ is not projective.

In Step 1 in the proof of [Fn4, Theorem 6.2], $S$ is a compact smooth analytic surface with $\kappa\left(S, K_{S}\right)=0$ and there are no $(-1)$-curves on $S$. Since $S$ is in Fujiki's class $\mathcal{C}$, the first Betti number $b_{1}(S)$ of $S$ is even. Therefore, by the Enriques-Kodaira classification, $S$ is a $K 3$ surface or a complex torus (see Lemma 7.4). Then, by Lemmas 7.5 and 7.6, we have $\kappa\left(X, K_{X}+\Delta\right)=\kappa\left(S, K_{S}+\Delta_{S}\right)=\kappa(S, Z) \geqslant 1$ and get a contradiction. This means that Step 1 in the proof of [Fn4, Theorem 6.2] works when $S$ is not projective.

Therefore, $K_{X}+\Delta$ is always semiample. This is what we wanted.

\section{§8. Contraction theorem for log canonical surfaces}

In this section, we discuss a contraction theorem for log canonical surfaces. Note that compact log canonical surfaces are not necessarily $\mathbb{Q}$-factorial. Therefore, we need Mumford's intersection theory (see [Mu1], [Ma, Remark 4-6-3], and [S]).

Definition 8.1. (Mumford's intersection theory) Let $X$ be a normal analytic surface, and let $\pi: Y \rightarrow X$ be a resolution. Let $\operatorname{Exc}(\pi)=\sum_{i} E_{i}$ be the irreducible decomposition of the exceptional curve of $\pi$. Let $D$ be a $\mathbb{Q}$-divisor on $X$. Then we can define the inverse image $\pi^{*} D$ as

$$
\pi^{*} D=D^{\dagger}+\sum_{i} \alpha_{i} E_{i}
$$

where $D^{\dagger}$ is the strict transform of $D$ by $\pi$ and the rational numbers $\alpha_{i}$ are uniquely determined by the following linear equations:

$$
D^{\dagger} \cdot E_{j}+\sum_{i} \alpha_{i} E_{i} \cdot E_{j}=0
$$

for every $j$. We call $\pi^{*} D$ the pull-back of $D$ in the sense of Mumford. Of course, $\pi^{*} D$ coincides with the usual one when $D$ is $\mathbb{Q}$-Cartier.

From now on, we further assume that $X$ is compact. The intersection number $D \cdot D^{\prime}$ (in the sense of Mumford) is defined to be the rational number $\left(\pi^{*} D\right) \cdot\left(\pi^{*} D^{\prime}\right)$, where $D$ and $D^{\prime}$ are $\mathbb{Q}$-divisors on $X$. We can easily see that $D \cdot D^{\prime}$ is well defined. We note that it coincides with the usual one when $D$ or $D^{\prime}$ is $\mathbb{Q}$-Cartier. 
Let us recall some definitions and basic properties of surface singularities for the reader's convenience.

Definition 8.2. (Numerically log canonical and numerically dlt; see [KM, Notation 4.1]) Let $X$ be a normal analytic surface, and let $\Delta$ be a $\mathbb{Q}$-divisor on $X$. Let $f: Y \rightarrow U \subset X$ be a proper bimeromorphic morphism from a smooth surface $Y$ to an open set $U$ of $X$. Then we can define $f^{*}\left(K_{U}+\left.\Delta\right|_{U}\right)$ in the sense of Mumford (see Definition 8.1) without assuming that $K_{U}+\left.\Delta\right|_{U}$ is $\mathbb{Q}$-Cartier. Thus we can always write

$$
K_{Y}=f^{*}\left(K_{U}+\left.\Delta\right|_{U}\right)+\sum_{E_{i}} a\left(E_{i}, X, \Delta\right) E_{i}
$$

such that $f_{*}\left(\sum_{E_{i}} a\left(E_{i}, X, \Delta\right) E_{i}\right)=-\left.\Delta\right|_{U}$. If $\Delta$ is effective and $a\left(E_{i}, X, \Delta\right) \geqslant-1$ for every exceptional curve $E_{i}$ and $f: Y \rightarrow U \subset X$, then we say that $(X, \Delta)$ is numerically log canonical. We say that $(X, \Delta)$ is numerically dlt if $(X, \Delta)$ is numerically log canonical and there exists a finite set $Z \subset X$ such that $X \backslash Z$ is smooth, Supp $\left.\Delta\right|_{X \backslash Z}$ is a simple normal crossing divisor on $X \backslash Z$, and $a(E, X, \Delta)>-1$ for every exceptional curve $E$ which maps to $Z$. It is well known that if $(X, \Delta)$ is numerically $\log$ canonical, then $K_{X}+\Delta$ is $\mathbb{Q}$-Cartier (see [Fn4, Proposition 3.5] and [Ma, Remark 4-6-3]). Moreover, if $(X, \Delta)$ is numerically dlt, then $X$ has only rational singularities (see [KM, Theorem 4.12]).

REMARK 8.3. In Definition 8.2, we only require that Supp $\left.\Delta\right|_{X \backslash Z}$ is a simple normal crossing divisor on $X \backslash Z$ in the classical topology. So it permits some irreducible component of Supp $\left.\Delta\right|_{X \backslash Z}$ to have nodal singularities. Therefore, our definition does not coincide with [KM, Notation 4.1] when $X$ is an algebraic surface. However, since we are mainly interested in local analytic properties of singularities of pairs $(X, \Delta)$, this difference causes no subtle problems.

We need the following contraction theorem for log canonical surfaces in Sections 9 and 10.

Theorem 8.4. (Contraction theorem for log canonical surfaces; see [Fn6, Theorem 4.1]) Let $(X, \Delta)$ be a compact log canonical surface, and let $C$ be an irreducible curve on $X$ such that $-\left(K_{X}+\Delta\right) \cdot C>0$ and $C^{2}<0$, where $C^{2}$ is the self-intersection number of $C$ in the sense of Mumford (see Definition 8.1). Then we have a projective bimeromorphic morphism $\varphi: X \rightarrow Y$ onto a normal surface $Y$ such that $\operatorname{Exc}(\varphi)=C \simeq \mathbb{P}^{1}$ and that $C$ passes through no nonrational singular points of $X$, that is, $X$ has only rational singularities in a neighborhood of $C$. In particular, $C$ is $\mathbb{Q}$-Cartier. Moreover, $\left(Y, \Delta_{Y}\right)$ is log canonical with $\Delta_{Y}:=\varphi_{*} \Delta$.

Proof. By Sakai's contraction theorem (see [S, Theorem (1.2)]), we have a bimeromorphic morphism $\varphi: X \rightarrow Y$, which contracts $C$ to a normal point $P \in Y$. Since $-\left(K_{X}+\Delta\right) \cdot C>$ $0,\left(Y, \Delta_{Y}\right)$ is numerically dlt in a neighborhood of $P$ by the negativity lemma. Therefore, $K_{Y}+\Delta_{Y}$ is $\mathbb{Q}$-Cartier and $Y$ has only rational singularities in a neighborhood of $P$. Of course, $\left(Y, \Delta_{Y}\right)$ is a compact log canonical surface. By Theorem $11.3, R^{i} \varphi_{*} \mathcal{O}_{X}=0$ for every $i>0$. Thus, $X$ has only rational singularities in a neighborhood of $C$ by Lemma 2.8. In particular, $C$ is $\mathbb{Q}$-Cartier (see [Nk, Chapter II, 2.12. Lemma]). Since $R^{1} \varphi_{*} \mathcal{O}_{X}=0$, we can easily check that $C \simeq \mathbb{P}^{1}$ as in Claim 1 of the proof of Theorem 5.1. We see that $\varphi$ is projective; $-\left(K_{X}+\Delta\right)$ and $-C$ are $\varphi$-ample by construction.

We close this section with simple but very important remarks. 
REMARK 8.5. (Extremal rays) Theorem 8.4 says that $X$ has only rational singularities in a neighborhood of the exceptional curve $C$ and then $C$ is automatically $\mathbb{Q}$-Cartier. Therefore, if $X$ is projective, then $C$ spans a $\left(K_{X}+\Delta\right)$-negative extremal ray $R$ of $\overline{\mathrm{NE}}(X)$ in the usual sense (see Lemma 5.2). Thus the contraction $\varphi$ in Theorem 8.4 is nothing but the usual contraction morphism associated with the extremal ray $R$. In particular, $Y$ is also projective when so is $X$.

REMARK 8.6. (Termination of contractions) Assume that $X$ is Moishezon. We consider a sequence of contraction morphisms as in Theorem 8.4,

$$
(X, \Delta)=:\left(X_{0}, \Delta_{0}\right) \stackrel{\varphi_{0}}{\longrightarrow}\left(X_{1}, \Delta_{1}\right) \stackrel{\varphi_{1}}{\longrightarrow} \cdots \stackrel{\varphi_{i-1}}{\longrightarrow}\left(X_{i}, \Delta_{i}\right) \stackrel{\varphi_{i}}{\longrightarrow} \cdots,
$$

starting from a log canonical surface $\left(X_{0}, \Delta_{0}\right):=(X, \Delta)$. Let $C_{i}$ be the $\varphi_{i}$-exceptional curve for every $i$. By Theorem 8.4, $C_{i}$ is $\mathbb{Q}$-Cartier for every $i$. Then we can easily see that $C_{0}$, $\varphi_{0}^{*} C_{1}, \ldots, \varphi_{0}^{*} \cdots \varphi_{i-1}^{*} C_{i}, \ldots$ are linearly independent in $N^{1}(X)$. Therefore, the sequence must terminate since $\rho(X)<\infty$.

\section{§9. Log canonical surfaces in Fujiki's class $\mathcal{C}$ with negative Kodaira dimension}

The main purpose of this section is to prove the following theorem.

TheOrem 9.1. (see Theorem 1.3) Let $(X, \Delta)$ be a log canonical surface in Fujiki's class $\mathcal{C}$. Assume that $\kappa\left(X, K_{X}+\Delta\right)=-\infty$ holds. Then $X$ is projective.

Let us recall the following well-known lemma for the reader's convenience (see [Ma, Remark 4-6-29]).

Lemma 9.2. Let $(X, \Delta)$ be a log canonical surface. Assume that $P \in X$ is not a rational singularity. Then $P \notin \operatorname{Supp} \Delta$ and $X$ is Gorenstein at $P$.

Sketch of Proof. If $P \in \operatorname{Supp} \Delta$, then $(X, 0)$ is numerically dlt in a neighborhood of $P$. In particular, $X$ has only rational singularities in a neighborhood of $P$. Therefore, we have $P \notin \operatorname{Supp} \Delta$. By the classification of two-dimensional log canonical singularities (see [KM, Theorem 4.7] and [Ma, Theorem 4-6-28]), $P \in X$ is a simple elliptic singularity or a cusp singularity (see [KM, Note 4.8] and [Ma, Theorem 4-6-28]). We can check that all the other two-dimensional log canonical singularities are rational singularities (see [Ma, Remark 4-629]). Therefore, $X$ is Gorenstein at $P$.

Let us start the proof of Theorem 9.1.

Proof of Theorem 9.1. We divide the proof into several small steps.

Step 1. In this step, we will prove that $X$ is Moishezon, that is, the algebraic dimension $a(X)$ of $X$ is two.

Let $f: Y \rightarrow X$ be the minimal resolution of $X$ with $K_{Y}+\Delta_{Y}:=f^{*}\left(K_{X}+\Delta\right)$. Then $\left(Y, \Delta_{Y}\right)$ is $\log$ canonical since so is $(X, \Delta)$ by assumption. By applying Lemma 4.3 to $\left(Y, \Delta_{Y}\right)$, we obtain that $Y$ is a smooth projective surface. This implies that $X$ is Moishezon, that is, the algebraic dimension $a(X)$ of $X$ is two.

Step 2. If $X$ has only rational singularities, then $X$ is $\mathbb{Q}$-factorial (see Lemma 3.10). In this case, by Lemma $4.1, X$ is projective since we have already known that $X$ is Moishezon in Step 1. 
Therefore, from now on, we may assume that $X$ has at least one nonrational singular point.

Step 3. By applying Theorem 8.4 finitely many times, we may assume that if $C$ is an irreducible curve on $X$ with $-\left(K_{X}+\Delta\right) \cdot C>0$, then $C^{2} \geqslant 0$ holds (see Remark 8.6).

Step 4. Let $g: Z \rightarrow X$ be the minimal resolution of nonrational singularities of $X$. Then we get the following commutative diagram:

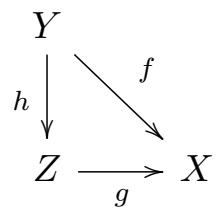

Since $Z$ has only rational singularities by construction, we see that $Z$ is $\mathbb{Q}$-factorial (see Lemma 3.10). Therefore, $Z$ is projective by Lemma 4.1 since $Z$ is Moishezon (see Step 1). We put $K_{Z}+\Delta_{Z}:=g^{*}\left(K_{X}+\Delta\right)$. Then $\Delta_{Z}$ is effective by the negativity lemma. Of course, $\left(Z, \Delta_{Z}\right)$ is $\log$ canonical. More precisely, by Lemma 9.2 , we have $\Delta_{Z}=\sum_{i} E_{i}+g_{*}^{-1} \Delta$, where $\operatorname{Exc}(g)=\sum_{i} E_{i}$. Since $K_{Z}+\Delta_{Z}$ never becomes nef by $\kappa\left(Z, K_{Z}+\Delta_{Z}\right)=\kappa\left(X, K_{X}+\Delta\right)=$ $-\infty$ (see Theorem 7.1$)$, there exists a $\left(K_{Z}+\Delta_{Z}\right)$-negative extremal ray $R=\mathbb{R}_{\geqslant 0}[\bar{C}]$ of $\overline{\mathrm{NE}}(Z)$, where $\bar{C}$ is an irreducible rational curve on $Z$.

Step 5. In this step, we will prove the following claim.

Claim 2. The self-intersection number $\bar{C}^{2}$ of $\bar{C}$ is nonnegative, where $\bar{C}$ is a $\left(K_{Z}+\Delta_{Z}\right)$ negative extremal rational curve as in Step 4.

Proof of Claim 2. We assume that $\bar{C}^{2}<0$ holds. Since $-\left(K_{Z}+\Delta_{Z}\right) \cdot \bar{C}>0, \bar{C}$ is not $g$-exceptional. If $C_{X}:=g_{*} \bar{C}$ is disjoint from nonrational singularities of $X$, then we have $\left(C_{X}\right)^{2}<0$ and $-\left(K_{X}+\Delta\right) \cdot C_{X}>0$ because $g$ is an isomorphism in a neighborhood of $\bar{C}$. This is a contradiction by Step 3 . Therefore, $C_{X}$ passes though at least one nonrational singular point $P$ of $X$. This implies that $C_{X} \not \subset \operatorname{Supp} \Delta$ by Lemma 9.2. Let $C_{Y}$ be the strict transform of $\bar{C}$ on $Y$. Then we can easily see that $\left(C_{Y}\right)^{2}<0$ holds and that $C_{Y}$ is not contained in Supp $\Delta_{Y}$. Thus we obtain that $-K_{Y} \cdot C_{Y} \geqslant-\left(K_{Y}+\Delta_{Y}\right) \cdot C_{Y}=-\left(K_{Z}+\right.$ $\left.\Delta_{Z}\right) \cdot \bar{C}>0$. This means that $C_{Y}$ is a $(-1)$-curve on $Y$. In particular, $-K_{Y} \cdot C_{Y}=1$. On the other hand, $\Delta_{Y} \cdot C_{Y} \geqslant 1$ since $C_{X}$ passes through a nonrational singular point $P$ and the reduced part of $\Delta_{Y}$ contains $f^{-1}(P)$. Thus we have $-\left(K_{Y}+\Delta_{Y}\right) \cdot C_{Y}=1-\Delta_{Y} \cdot C_{Y} \leqslant 0$. This is a contradiction. Therefore, $\bar{C}^{2} \geqslant 0$ holds.

Therefore, every $\left(K_{Z}+\Delta_{Z}\right)$-negative extremal ray is spanned by an irreducible rational curve $\bar{C}$ with $\bar{C}^{2} \geqslant 0$.

We will treat the case where $\bar{C}^{2}>0$ and $\bar{C}^{2}=0$ in Steps 6 and 7 , respectively.

Step 6. We assume that there exists a $\left(K_{Z}+\Delta_{Z}\right)$-negative extremal ray $R$ of $\overline{\mathrm{NE}}(Z)$ spanned by an irreducible rational curve $\bar{C}$ with $\bar{C}^{2}>0$. In this case, the numerical equivalence class of $\bar{C}$ is an interior point of $\overline{\mathrm{NE}}(Z)$ (see [KM, Corollary 1.21]) and it also generates an extremal ray of $\overline{\mathrm{NE}}(Z)$. Therefore, it is easy to see that $\rho(Z)=1$ and $-\left(K_{Z}+\Delta_{Z}\right)$ is ample. This is a contradiction because $-\left(K_{Z}+\Delta_{Z}\right) \cdot E=0$ for every $g$ exceptional curve $E$ on $Z$. Thus, this case does not happen. 
Step 7. Hence we may assume that there exists a $\left(K_{Z}+\Delta_{Z}\right)$-negative extremal ray $R$ spanned by an irreducible rational curve $\bar{C}$ with $\bar{C}^{2}=0$. Then there exists a surjective morphism $\varphi_{R}: Z \rightarrow W$ onto a smooth projective curve $W$ such that $\rho(Z / W)=1$ and $-\left(K_{Z}+\Delta_{Z}\right)$ is $\varphi_{R}$-ample, that is, $\varphi_{R}: Z \rightarrow W$ is a Mori fiber space. Without loss of generality, we may assume that $\bar{C}$ is a general fiber of $\varphi_{R}$. Since the self-intersection number of any irreducible component of $\operatorname{Exc}(g)$ is negative, every irreducible component of $\operatorname{Exc}(g)$ is dominant onto $W$ by $\varphi_{R}$. Since $-K_{Z} \cdot \bar{C}=2$ and $\Delta_{Z} \geqslant \sum_{i} E_{i}=\operatorname{Exc}(g)$, we can check that $E:=\operatorname{Exc}(g)$ is an irreducible curve such that $\left.\varphi_{R}\right|_{E}: E \rightarrow W$ is an isomorphism. By the classification of two-dimensional log canonical singularities (see [KM, Theorem 4.7] and [Ma, Theorem 4-6-28]), $E$ is an elliptic curve and $P$ is a simple elliptic singularity.

Claim 3. $-K_{X}$ is ample, that is, $X$ is Fano. In particular, $X$ is projective.

Proof of Claim 3. Since $\varphi_{R}: Z \rightarrow W$ is a Mori fiber space and $-\left(K_{Z}+E\right) \cdot \bar{C}=E$. $\bar{C}=1$, we obtain that $-\left(K_{Z}+E\right)$ is $\mathbb{Q}$-linearly equivalent to $E+\varphi_{R}^{*} D$, where $D$ is some $\mathbb{Q}$-divisor on $W$. We note that $\operatorname{deg} D=-E^{2}>0$ since $\left(E+\varphi_{R}^{*} D\right) \cdot E=-\left(K_{Z}+E\right) \cdot E=$ 0 . Thus, we have $\kappa\left(Z,-\left(K_{Z}+E\right)\right) \geqslant 0$. Since $K_{Z}+E=g^{*} K_{X}$, we obtain $\kappa\left(X,-K_{X}\right)=$ $\kappa\left(Z,-\left(K_{Z}+E\right)\right) \geqslant 0$. We take any irreducible curve $C$ on $X$. Let $C^{\prime}$ be the strict transform of $C$ on $Z$. Then $-K_{X} \cdot C=-\left(K_{Z}+E\right) \cdot C^{\prime}=\left(E+\varphi_{R}^{*} D\right) \cdot C^{\prime}>0$ since $C^{\prime} \neq E$. Thus, we obtain that $-K_{X}$ is ample by Nakai-Moishezon's ampleness criterion for algebraic spaces (see $[\mathrm{P},(1.4)$ Theorem]).

Thus we see that $X$ is projective when $X$ has at least one nonrational singular point.

Therefore, we obtain that $X$ is always projective if $(X, \Delta)$ is a log canonical surface in Fujiki's class $\mathcal{C}$ with $\kappa\left(X, K_{X}+\Delta\right)=-\infty$.

REMARK 9.3. Let $(X, \Delta)$ be a $\log$ canonical surface in Fujiki's class $\mathcal{C}$ with $\kappa\left(X, K_{X}+\right.$ $\Delta)=-\infty$. The proof of Theorem 9.1 shows that if $X$ has nonrational singularities, then $X$ has one simple elliptic singularity and no cusp singularities.

By Theorem 9.1, we can freely apply the minimal model theory of projective log canonical surfaces established in [Fn4] to log canonical surfaces in Fujiki's class $\mathcal{C}$ with negative Kodaira dimension.

REMARK 9.4. We can construct a complete nonprojective log canonical algebraic surface $(X, \Delta)$ with $\kappa\left(X, K_{X}+\Delta\right) \geqslant 0$. For some examples, see Section 12 . Therefore, the assumption $\kappa\left(X, K_{X}+\Delta\right)=-\infty$ is indispensable in Theorem 9.1.

\section{$\S 10$. Proof of Theorem 1.5}

In this section, we prove Theorem 1.5, that is, the minimal model theory for log canonical surfaces in Fujiki's class $\mathcal{C}$. We give a detailed proof for the reader's convenience although it is essentially the same as that of Theorem 1.1.

Proof of Theorem 1.5. If $\left(K_{X}+\Delta\right) \cdot C \geqslant 0$ for every curve $C$ on $X$, then $K_{X}+\Delta$ is semiample by Corollary 7.7. So $(X, \Delta)$ is itself a good minimal model of $(X, \Delta)$. Therefore, we may assume that $\left(K_{X}+\Delta\right) \cdot C<0$ for some curve $C$ on $X$. If $X$ is projective, then we can run the minimal model program for projective log canonical surfaces and finally get a good minimal model or a Mori fiber space (see [Fn4]). Thus we may assume that $X$ is not projective. By Theorem 9.1 , we obtain $\kappa\left(X, K_{X}+\Delta\right) \geqslant 0$. Therefore, we have an effective Cartier divisor $D \in\left|m\left(K_{X}+\Delta\right)\right|$ for some positive divisible integer $m$. Since 
$\left(K_{X}+\Delta\right) \cdot C<0$ for some curve $C$ on $X, C$ is an irreducible component of Supp $D$ such that the self-intersection number $C^{2}$ is negative. We apply the contraction theorem, Theorem 8.4. Since there are only finitely many irreducible components of Supp $D$, we finally get $\left(X^{*}, \Delta^{*}\right)$ such that $K_{X^{*}}+\Delta^{*}$ is semiample after finitely many contractions.

Since $R^{1} \varphi_{i_{*}} \mathcal{O}_{X_{i}}=0$ by Theorem $11.3, X_{i}$ has only rational singularities if and only if so does $X_{i+1}$ by Lemma 2.8. Therefore, we have (2).

Since $\varphi_{i}$ is projective by construction, $X_{i}$ is projective when so is $X_{i+1}$. If $X_{i}$ is projective, then $\varphi_{i}$ is the usual contraction morphism associated with a $\left(K_{X_{i}}+\Delta_{i}\right)$-negative extremal ray (see Remark 8.5). This implies that $X_{i+1}$ is also projective. Thus we have (1).

Since $\operatorname{Exc}\left(\varphi_{i}\right) \simeq \mathbb{P}^{1}, C_{i}=\operatorname{Exc}\left(\varphi_{i}\right)$ is $\mathbb{Q}$-Cartier, and $-C_{i}$ is $\varphi_{i}$-ample, we can easily check (3) by Theorem 3.11 .

\section{§11. Appendix: Vanishing theorems}

In this section, we quickly explain some vanishing theorems for the reader's convenience. Fortunately, we do not need difficult analytic methods. They follow from elementary arguments.

Let us start with the following vanishing theorem. We learned it from $[\mathrm{KK}]$ (see $[\mathrm{K} 2$, Theorem 10.4]).

THEOREM 11.1. (Relative vanishing theorem) Let $\varphi: V \rightarrow W$ be a proper bimeromorphic morphism from a smooth analytic surface to a normal analytic surface $W$. Assume that there exists a point $P \in W$ such that $\varphi$ is an isomorphism over $W \backslash P$. Let $\operatorname{Exc}(\varphi)=\sum_{i} E_{i}$ be the irreducible decomposition of the $\varphi$-exceptional locus $\operatorname{Exc}(\varphi)$. Let $\mathcal{L}$ be a line bundle on $V$, let $N$ be a $\mathbb{Q}$-divisor on $V$, and let $E=\sum_{i} b_{i} E_{i}$ be an effective $\mathbb{Q}$-divisor on $V$. Assume that $N \cdot E_{i} \geqslant 0$ and $\mathcal{L} \cdot E_{i}=\left(K_{V}+E+N\right) \cdot E_{i}$ hold for every $i$. We further assume that one of the following conditions holds.

(1) $0 \leqslant b_{i}<1$ for every $i$.

(2) $0<b_{i} \leqslant 1$ for every $i$ and there exists some $j$ such that $b_{j} \neq 1$.

(3) $0<b_{i} \leqslant 1$ for every $i$ and there exists some $j$ such that $N \cdot E_{j}>0$.

Then $R^{i} \varphi_{*} \mathcal{L}=0$ holds for every $i>0$.

Theorem 11.1(1) is a special case of the Kawamata-Viehweg vanishing theorem. Note that condition (1) is most useful.

REMARK 11.2. In Theorem 11.1, it is sufficient to assume that $N$ is a $\mathbb{Q}$-line bundle on $V$, that is, a finite $\mathbb{Q}$-linear combination of some line bundles on $V$ such that $N \cdot E_{i} \geqslant 0$ and $\mathcal{L} \cdot E_{i}=\left(K_{V}+E\right) \cdot E_{i}+N \cdot E_{i}$ hold for every $i$.

Proof of Theorem 11.1. Let us consider $Z=\sum_{i} r_{i} E_{i}$, where $r_{i}$ is a nonnegative integer for every $i$. Although [K2, Theorem 10.4] is formulated and proved for two-dimensional regular schemes, the proof works for two-dimensional complex manifolds. Therefore, by the proof of [K2, Theorem 10.4], we obtain $H^{1}\left(Z, \mathcal{L} \otimes \mathcal{O}_{Z}\right)=0$ (see also [KK, 2.2.1 Theorem]). Hence, by using the theorem on formal functions for proper morphisms between analytic spaces (see [BS, Chapter VI, Corollary 4.7]), we get $R^{i} \varphi_{*} \mathcal{L}=0$ for every $i>0$ (see also [KK, 2.2.5 Corollary]). 
As an application of Theorem 11.1, we can prove the following vanishing theorem, which is a Kawamata-Viehweg vanishing theorem for proper bimeromorphic morphisms between surfaces.

THEOREM 11.3. Let $X$ be a normal analytic surface, and let $\Delta$ be an effective $\mathbb{Q}$-divisor on $X$ such that $K_{X}+\Delta$ is $\mathbb{Q}$-Cartier. Let $f: X \rightarrow Y$ be a proper bimeromorphic morphism onto a normal analytic surface $Y$. Let $\mathcal{L}$ be a line bundle on $X$, and let $D$ be a $\mathbb{Q}$-Cartier Weil divisor on $X$. Assume that one of the following conditions holds.

(1) $\mathcal{L} \cdot C+\left(D-\left(K_{X}+\Delta\right)\right) \cdot C>0$ for every $f$-exceptional curve $C$ on $X$ and the coeffcients of $\Delta$ are less than or equal to one.

(2) $\mathcal{L} \cdot C+\left(D-\left(K_{X}+\Delta\right)\right) \cdot C \geqslant 0$ for every $f$-exceptional curve $C$ on $X$ and the coeffcients of $\Delta$ are less than one.

Then $R^{i} f_{*}\left(\mathcal{L} \otimes \mathcal{O}_{X}(D)\right)=0$ holds for every $i>0$.

Let us prove Theorem 11.3. The following proof is essentially the same as that of [FT, Theorem 6.2].

Proof of Theorem 11.3. We divide the proof into small steps.

Step 1. Without loss of generality, we can freely shrink $Y$ and assume that $Y$ is a small relatively compact Stein open subset of normal analytic surface. We may further assume that $f$ is an isomorphism outside $P \in Y$ and $f^{-1}(P)$ is one-dimensional.

Step 2. When $\lfloor\Delta\rfloor \neq 0$, we can take an $f$-ample Cartier divisor $H$ (see (1)). Then we can find an effective $\mathbb{Q}$-divisor $\Delta^{\prime}$ on $X$ such that $\left\lfloor\Delta^{\prime}\right\rfloor=0$ and that $\Delta^{\prime}$ is $\mathbb{Q}$-linearly equivalent to $\Delta+\varepsilon H$ for some $0<\varepsilon \ll 1$. More precisely, we take a general member $B$ of $\lfloor\lfloor\rfloor\rfloor+m H \mid$ for some large positive integer $m$ and put

$$
\Delta^{\prime}=\Delta-\frac{1}{m}\lfloor\Delta\rfloor+\frac{1}{m} B .
$$

By replacing $\Delta$ with $\Delta^{\prime}$, we can always assume that $\lfloor\Delta\rfloor=0$.

Step 3. Let $\varphi: Z \rightarrow X$ be the minimal resolution of $X$. We set $K_{Z}+\Delta_{Z}:=\varphi^{*}\left(K_{X}+\right.$ $\Delta)$. We note that $\Delta_{Z}$ is effective by the negativity lemma. We note that

$$
\varphi^{*} \mathcal{L}+\left\lceil\varphi^{*} D\right\rceil-\left(K_{Z}+\Delta_{Z}+\left\{-\varphi^{*} D\right\}\right)=\varphi^{*}\left(\mathcal{L}+D-\left(K_{X}+\Delta\right)\right) .
$$

We put $\Theta:=\Delta_{Z}+\left\{-\varphi^{*} D\right\}$. Then

$$
\varphi^{*} \mathcal{L}+\left\lceil\varphi^{*} D\right\rceil-\lfloor\Theta\rfloor=\left(K_{Z}+\{\Theta\}\right)+\varphi^{*}\left(\mathcal{L}+D-\left(K_{X}+\Delta\right)\right) .
$$

We note that we can write $\{\Theta\}=E+M$, where $E$ is a $\varphi$-exceptional effective $\mathbb{Q}$-divisor with $\lfloor E\rfloor=0$ and $M$ is an effective $\mathbb{Q}$-divisor such that every irreducible component of $M$ is not $\varphi$-exceptional. Let us consider

$$
\begin{aligned}
0 & \rightarrow \varphi^{*} \mathcal{L} \otimes \mathcal{O}_{Z}\left(\left\lceil\varphi^{*} D\right\rceil-\lfloor\Theta\rfloor\right) \rightarrow \varphi^{*} \mathcal{L} \otimes \mathcal{O}_{Z}\left(\left\lceil\varphi^{*} D\right\rceil\right) \\
& \rightarrow \varphi^{*} \mathcal{L} \otimes \mathcal{O}_{\lfloor\Theta\rfloor}\left(\left\lceil\varphi^{*} D\right\rceil\right) \rightarrow 0 .
\end{aligned}
$$

By Theorem 11.1, we have

$$
R^{1} \varphi_{*}\left(\varphi^{*} \mathcal{L} \otimes \mathcal{O}_{Z}\left(\left\lceil\varphi^{*} D\right\rceil-\lfloor\Theta\rfloor\right)\right)=0 .
$$


Therefore, we get the following short exact sequence:

$$
\begin{aligned}
0 & \rightarrow \mathcal{L} \otimes \varphi_{*} \mathcal{O}_{Z}\left(\left\lceil\varphi^{*} D\right\rceil-\lfloor\Theta\rfloor\right) \rightarrow \mathcal{L} \otimes \varphi_{*} \mathcal{O}_{Z}\left(\left\lceil\varphi^{*} D\right\rceil\right) \\
& \rightarrow \mathcal{L} \otimes \varphi_{*} \mathcal{O}_{\lfloor\Theta\rfloor}\left(\left\lceil\varphi^{*} D\right\rceil\right) \rightarrow 0 .
\end{aligned}
$$

By construction, $\lfloor\Theta\rfloor$ is $\varphi$-exceptional. Therefore, $\mathcal{L} \otimes \varphi_{*} \mathcal{O}_{\lfloor\Theta\rfloor}\left(\left\lceil\varphi^{*} D\right\rceil\right)$ is a skyscraper sheaf on $X$. Thus we obtain the following surjection:

$$
R^{1} f_{*}\left(\mathcal{L} \otimes \varphi_{*} \mathcal{O}_{Z}\left(\left\lceil\varphi^{*} D\right\rceil-\lfloor\Theta\rfloor\right)\right) \rightarrow R^{1} f_{*}\left(\mathcal{L} \otimes \mathcal{O}_{X}(D)\right) \rightarrow 0
$$

since $\varphi_{*} \mathcal{O}_{Z}\left(\left\lceil\varphi^{*} D\right\rceil\right) \simeq \mathcal{O}_{X}(D)$. By the Leray spectral sequence, we have

$$
R^{1} f_{*}\left(\mathcal{L} \otimes \varphi_{*} \mathcal{O}_{Z}\left(\left\lceil\varphi^{*} D\right\rceil-\lfloor\Theta\rfloor\right)\right) \subset R^{1}(f \circ \varphi)_{*}\left(\varphi^{*} \mathcal{L} \otimes \mathcal{O}_{Z}\left(\left\lceil\varphi^{*} D\right\rceil-\lfloor\Theta\rfloor\right)\right) .
$$

As before, we can write $\{\Theta\}=E^{\prime}+M^{\prime}$, where $E^{\prime}$ is a $f \circ \varphi$-exceptional effective $\mathbb{Q}$-divisor with $\left\lfloor E^{\prime}\right\rfloor=0$ and $M^{\prime}$ is an effective $\mathbb{Q}$-divisor such that every irreducible component of $M^{\prime}$ is not $f \circ \varphi$-exceptional. By Theorem 11.1, we know that

$$
R^{1}(f \circ \varphi)_{*}\left(\varphi^{*} \mathcal{L} \otimes \mathcal{O}_{Z}\left(\left\lceil\varphi^{*} D\right\rceil-\lfloor\Theta\rfloor\right)\right)=0 .
$$

This implies that

$$
R^{1} f_{*}\left(\mathcal{L} \otimes \varphi_{*} \mathcal{O}_{Z}\left(\left\lceil\varphi^{*} D\right\rceil-\lfloor\Theta\rfloor\right)\right)=0
$$

by (11.2). By surjection (11.1), we get $R^{1} f_{*}\left(\mathcal{L} \otimes \mathcal{O}_{X}(D)\right)=0$.

Step 4. Since $f^{-1}(P)$ is one-dimensional, $R^{i} f_{*}\left(\mathcal{L} \otimes \mathcal{O}_{X}(D)\right)=0$ for every $i>2$ by the theorem on formal functions for proper morphisms between analytic spaces (see [BS, Chapter VI, Corollary 4.7]).

Therefore, we have $R^{i} f_{*}\left(\mathcal{L} \otimes \mathcal{O}_{X}(D)\right)=0$ for every $i>0$.

We close this section with an obvious remark.

REMARK 11.4. Theorems 11.1 and 11.3 can be formulated and proved easily for proper birational morphisms between algebraic surfaces defined over any algebraically closed field. Therefore, we can formulate and prove Theorem 3.11 for projective birational morphisms between (not necessarily complete) algebraic surfaces defined over any algebraically closed field. This is because the proof of Theorem 3.11 only needs the vanishing theorem, Theorem 11.3.

\section{§12. Appendix: Complete nonprojective algebraic surfaces}

In this section, we construct some examples of complete nonprojective log canonical algebraic surfaces. From Example 12.1 to Example 12.5, we will work over $\mathbb{C}$, the complex number field.

Let us start with Kollár's example. Although it is not stated explicitly in [K1], it does not satisfy Kleiman's ampleness criterion. We note that the arguments in Example 12.3 help the reader understand Example 12.1. Therefore, we do not explain the details of Kollár's example. 
ExAmPle 12.1. ([K1, Aside 3.46]) In this example, we will freely use Kollár's notation in [K1, Aside 3.46]. In [K1, Aside 3.46], we assume that $C$ is an elliptic curve. Then the surface $S$ constructed in [K1, Aside 3.46] is a complete nonprojective algebraic surface with two simple elliptic singularities. In particular, $S$ is Gorenstein and log canonical. Let $C^{\prime}$ be the strict transform of $\{1\} \times C$ on $S$. Then we have $\operatorname{Pic}(S)=\mathbb{Z} \mathcal{O}_{S}\left(C^{\prime}\right)$. We can directly check that $\pi_{1}(S)=\{1\}$, that is, $S$ is simply connected, $K_{S} \sim 0, H^{1}\left(S, \mathcal{O}_{S}\right)=0$, and $H^{2}\left(S, \mathcal{O}_{S}\right)=\mathbb{C}$. Therefore, $S$ is a log canonical Calabi-Yau algebraic surface. Of course, we have $\kappa\left(S, K_{S}\right)=0$. Let $F$ be a general fiber of the second projection $\mathbb{P}^{1} \times C \rightarrow C$, and let $F^{\prime}$ be the strict transform of $F$ on $S$. Then $\overline{\mathrm{NE}}(S)=\mathbb{R}_{\geqslant 0}\left[F^{\prime}\right]$ holds. We note that $\mathcal{O}_{S}\left(C^{\prime}\right)$ is positive on $\overline{\mathrm{NE}}(S) \backslash\{0\}$. However, $C^{\prime}$ is nef but is not ample. This means that Kleiman's ampleness criterion does not hold for $S$.

Let us prepare an easy lemma.

LEMma 12.2. Let $C$ be a smooth projective curve, and let $\mathcal{A}$ be an ample line bundle on $C$. We consider $\pi: X:=\mathbb{P}_{C}\left(\mathcal{O}_{C} \oplus \mathcal{A}\right) \rightarrow C$. Then $\mathcal{O}_{X}(1) \simeq \mathcal{O}_{X}\left(C_{+}\right)$is semiample, where $C_{+}:=\mathbb{P}_{C}(\mathcal{A})$ is the positive section of $\pi$, and the complete linear system $\left|\mathcal{O}_{X}(m)\right|$ only contracts the negative section $C_{-}:=\mathbb{P}_{C}\left(\mathcal{O}_{C}\right)$ of $\pi$ to a point for some sufficiently large positive integer $m$.

Proof. We can easily check that $\mathcal{O}_{X}(1)$ is semiample (see [La, Lemma 2.3.2]). We note that $C_{-} \cdot \mathcal{O}_{X}(1)=0$ and $D \cdot \mathcal{O}_{X}(1)>0$ for every irreducible curve $D$ on $X$ with $D \neq C_{-}$. Therefore, the complete linear system $\left|\mathcal{O}_{X}(m)\right|$ contracts $C_{-}$only.

Let us construct complete nonprojective normal algebraic surfaces $S$ with $\operatorname{Pic}(S)=\{0\}$. The following construction was suggested by Kento Fujita.

EXAMPLE 12.3. Let $C$ be a smooth projective curve of genus $\geqslant 1$, and let $\mathcal{L}=\mathcal{O}_{C}(L)$ be a nontorsion element of $\operatorname{Pic}^{0}(C)$. We consider $\pi: V:=\mathbb{P}_{C}\left(\mathcal{O}_{C} \oplus \mathcal{L}\right) \rightarrow C$. Let $C_{1}$ (resp. $\left.C_{2}\right)$ be the section of $\pi$ corresponding to $\mathcal{O}_{C} \oplus \mathcal{L} \rightarrow \mathcal{O}_{C} \rightarrow 0$ (resp. $\mathcal{O}_{C} \oplus \mathcal{L} \rightarrow \mathcal{L} \rightarrow 0$ ). We note that $C_{2} \sim \pi^{*} L+C_{1}$ holds. We take an arbitrary point $P \in C$ and blow up $P_{1}$ and $P_{2}$, where $P_{i}:=\pi^{-1}(P) \cap C_{i}$ for $i=1,2$, to get $p: W \rightarrow V$. Let $C_{i}^{\prime}$ be the strict transform of $C_{i}$ on $W$ for $i=1,2$, and let $\ell$ be the strict transform of $\pi^{-1}(P)$ on $W$. Let $E_{i}$ denote the $(-1)$-curve on $W$ with $p\left(E_{i}\right)=P_{i}$ for $i=1,2$. We put

$$
\begin{gathered}
U_{1}:=W \backslash\left(C_{2}^{\prime} \cup E_{2} \cup \ell\right), \quad U_{2}:=W \backslash\left(C_{1}^{\prime} \cup E_{1} \cup \ell\right), \\
\text { and } U_{0}:=W \backslash\left(C_{1}^{\prime} \cup C_{2}^{\prime}\right) .
\end{gathered}
$$

We note that $C_{i}^{\prime} \subset U_{i}$ for $i=1,2$ and $\ell \subset U_{0}$ by construction. Then we can realize $U_{i}$ as a Zariski open subset of $\mathbb{P}_{C}\left(\mathcal{O}_{C} \oplus \mathcal{A}_{i}\right)$ with $\operatorname{deg} \mathcal{A}_{i}=1$ such that $C_{i}^{\prime}$ corresponds to the negative section $C_{-}$on $\mathbb{P}_{C}\left(\mathcal{O}_{C} \oplus \mathcal{A}_{i}\right)$ for $i=1,2$. By Lemma 12.2 , we can construct a projective birational morphism $U_{i} \rightarrow S_{i}$ onto a normal quasi-projective surface $S_{i}$ such that the exceptional locus is $C_{i}^{\prime}$ for $i=1,2$. Since $\ell$ is a $(-2)$-curve on a smooth projective surface $W$, we can construct a projective birational morphism $U_{0} \rightarrow S_{0}$ onto a normal quasi-projective surface $S_{0}$ such that the exceptional locus is $\ell$ and that $\ell$ is contracted to an $A_{1}$ singularity. Now $S_{0}, S_{1}$, and $S_{2}$ can be glued together to get a birational contraction morphism $q: W \rightarrow S$ onto a complete normal algebraic surface $S$, which only contracts $C_{1}^{\prime}$, $C_{2}^{\prime}$, and $\ell$. 
Claim 4. $\operatorname{Pic}(S)=\{0\}$ holds.

Proof of Claim 4. We take an arbitrary Cartier divisor $D$ on $S$. We put $\bar{D}:=q^{*} D$ and $D^{\dagger}:=p_{*} \bar{D}$. Then we can write $D^{\dagger} \sim \pi^{*} M+k C_{1}$ for some integer $k$ and some Cartier divisor $M$ on $C$ because $V$ is $\mathbb{P}_{C}\left(\mathcal{O}_{C} \oplus \mathcal{L}\right)$. We note that we can write

$$
p^{*} D^{\dagger}=\bar{D}+m_{1} E_{1}+m_{2} E_{2}
$$

for some integers $m_{1}$ and $m_{2}$. By construction, $\left.\mathcal{O}_{W}(\bar{D})\right|_{C_{i}^{\prime}}$ is trivial for $i=1,2$. Therefore, we have

$$
\begin{aligned}
\mathcal{O}_{C}(M) & \left.\left.\simeq \mathcal{O}_{V}\left(D^{\dagger}\right)\right|_{C_{2}} \simeq \mathcal{O}_{W}\left(p^{*} D^{\dagger}\right)\right|_{C_{2}^{\prime}} \\
& \left.\simeq \mathcal{O}_{W}\left(\bar{D}+m_{1} E_{1}+m_{2} E_{2}\right)\right|_{C_{2}^{\prime}} \simeq \mathcal{O}_{C}\left(m_{2} P\right)
\end{aligned}
$$

and

$$
\begin{aligned}
\mathcal{O}_{C}(M-k L) & \left.\left.\simeq \mathcal{O}_{V}\left(D^{\dagger}\right)\right|_{C_{1}} \simeq \mathcal{O}_{W}\left(p^{*} D^{\dagger}\right)\right|_{C_{1}^{\prime}} \\
& \left.\simeq \mathcal{O}_{W}\left(\bar{D}+m_{1} E_{1}+m_{2} E_{2}\right)\right|_{C_{1}^{\prime}} \simeq \mathcal{O}_{C}\left(m_{1} P\right) .
\end{aligned}
$$

This implies that $\mathcal{O}_{C}(k L) \simeq \mathcal{O}_{C}\left(\left(m_{2}-m_{1}\right) P\right)$. Since $\operatorname{deg} L=0$, we have $m_{1}=m_{2}$. By assumption, $\mathcal{L}=\mathcal{O}_{C}(L)$ is a nontorsion element of $\operatorname{Pic}^{0}(C)$. Thus, we get $k=0$. By construction again, we have $\bar{D} \cdot \ell=0$. Therefore,

$$
0=k=p^{*} D^{\dagger} \cdot \ell=\left(\bar{D}+m_{1} E_{1}+m_{2} E_{2}\right) \cdot \ell=m_{1}+m_{2}
$$

holds. Since we have already known that $m_{1}=m_{2}$ holds, we obtain that $m_{1}=m_{2}=0$. Thus, we have $\bar{D}=p^{*} D^{\dagger} \sim p^{*} \pi^{*} M$. Since $\left.\mathcal{O}_{W}(\bar{D})\right|_{C_{i}^{\prime}}$ is trivial for $i=1,2$, we see that $\mathcal{O}_{C}(M)$ is trivial. Therefore, we obtain $\bar{D} \sim 0$. So we get $D \sim 0$. This means that $\operatorname{Pic}(S)=\{0\}$.

By Claim 4, we see that $S$ is obviously nonprojective. By construction, we can see that $\pi_{1}(S)=\{1\}$, that is, $S$ is simply connected.

From now on, we assume that $C$ is an elliptic curve. Then $S$ has two simple elliptic singularities and one $A_{1}$ singularity. Moreover, $S$ is Gorenstein and $K_{S} \sim 0$. We note that $K_{V}+C_{1}+C_{2} \sim 0$ and $K_{W}+C_{1}^{\prime}+C_{2}^{\prime} \sim 0$ by construction. We can easily check that $H^{1}\left(S, \mathcal{O}_{S}\right)=0$ and $H^{2}\left(S, \mathcal{O}_{S}\right)=\mathbb{C}$ by using the Leray spectral sequence. Therefore, $S$ is a complete nonprojective log canonical Calabi-Yau algebraic surface with $\operatorname{Pic}(S)=\{0\}$.

From now on, by taking blow-ups of $S$ in Example 12.3, we construct complete nonprojective algebraic surfaces with a large Picard number.

EXAMPLE 12.4. Let $S$ be the surface constructed in Example 12.3, which is birationally equivalent to $\mathbb{P}_{C}\left(\mathcal{O}_{C} \oplus \mathcal{L}\right)$. As we saw above, $\operatorname{Pic}(S)=\{0\}$ holds. We take the minimal resolution $\mu: \widetilde{S} \rightarrow S$ of the unique $A_{1}$ singularity of $S$. Since $S$ is simply connected, so is $\widetilde{S}$. Let $E$ denote the exceptional curve of $\mu$. Of course, $E$ is the strict transform of $\ell$ in Example 12.3 and is a $(-2)$-curve on $\widetilde{S}$.

Claim 5. $\operatorname{Pic}(\widetilde{S})=\mathbb{Z}_{\widetilde{S}}(E)$ holds.

Proof of Claim 5. By construction, we see that $E \simeq \mathbb{P}^{1}$ and that $-E$ and $-\left(K_{\widetilde{S}}+E\right)$ are both $\mu$-ample. Thus, by Theorem 3.11 (see also Remark 11.4), we have $\operatorname{Pic}(\widetilde{S}) \otimes \mathbb{Q}=$ $\mathbb{Q} \mathcal{O}_{\widetilde{S}}(E)$ since $\operatorname{Pic}(S)=\{0\}$. Let $\mathcal{N}$ be a torsion element of $\operatorname{Pic}(\widetilde{S})$. Then we have $\mathcal{N} \cdot E=0$. 
Therefore, by Theorem 3.11 again, $\mathcal{N}$ is trivial. This means that $\operatorname{Pic}(\widetilde{S})$ is torsion-free. Thus, we can write $\operatorname{Pic}(\widetilde{S})=\mathbb{Z} \mathcal{M}$ for some line bundle $\mathcal{M}$ on $\widetilde{S}$. Therefore, there exists some integer $a$ such that $\mathcal{O}_{\widetilde{S}}(E) \simeq \mathcal{M}^{\otimes a}$. Since $E^{2}=-2$, we have $a E \cdot \mathcal{M}=-2$. Note that $E \cdot \mathcal{M}$ is an integer. If $a= \pm 2$, then $-2=E^{2}=a^{2} \mathcal{M}^{2}=4 \mathcal{M}^{2}$. This is a contradiction because $\mathcal{M}^{2}$ is an integer. Thus, we get $a= \pm 1$. This means that $\operatorname{Pic}(\widetilde{S})=\mathbb{Z} \mathcal{O}_{\widetilde{S}}(E)$.

By Claim 5 , we have $N^{1}(\widetilde{S})=\mathbb{R}$ and $\overline{\mathrm{NE}}(\widetilde{S})=N_{1}(\widetilde{S})=\mathbb{R}$. We note that $E^{2}=-2$ and that there exists a curve $G$ on $\widetilde{S}$ such that $G \cdot E>0$.

We further assume that $C$ is an elliptic curve. Then $K_{\widetilde{S}} \sim 0$ and $\widetilde{S}$ has only two simple elliptic singularities. Therefore, $\widetilde{S}$ is a complete nonprojective log canonical Calabi-Yau algebraic surface with $\overline{\mathrm{NE}}(\widetilde{S})=\mathbb{R}$.

ExAmple 12.5. Let $\widetilde{S}$ be the surface constructed in Example 12.4. We take finitely many smooth points $Q_{1}, Q_{2}, \ldots, Q_{\rho-1}$ of $\widetilde{S}$ with $\rho \geqslant 2$ such that $Q_{i} \neq Q_{j}$ for $i \neq j$ and $Q_{i} \notin E$ for every $i$. We blow up $Q_{1}, Q_{2}, \ldots, Q_{\rho-1}$ to get $\sigma: \bar{S} \rightarrow \widetilde{S}$. Let $B_{i}$ denote the $(-1)$ curve on $\bar{S}$ with $\sigma\left(B_{i}\right)=Q_{i}$ for every $i$. Then, by Theorem 3.11 (see also Remark 11.4), we can easily check that

$$
\operatorname{Pic}(\bar{S})=\mathbb{Z} \mathcal{O}_{\bar{S}}\left(\sigma^{*} E\right) \oplus \mathbb{Z} \mathcal{O}_{\bar{S}}\left(B_{1}\right) \oplus \cdots \oplus \mathbb{Z} \mathcal{O}_{\bar{S}}\left(B_{\rho-1}\right) .
$$

Claim 6. $\overline{\mathrm{NE}}(\bar{S})=N_{1}(\bar{S})=\mathbb{R}^{\rho}$ holds.

Proof of Claim 6. Let $D$ be a nef $\mathbb{R}$-Cartier $\mathbb{R}$-divisor on $\bar{S}$. It is sufficient to prove that $D$ is numerically trivial. By the above description of $\operatorname{Pic}(\bar{S})$, we can assume that $D=$ $b_{0} \sigma^{*} E+\sum_{i=1}^{\rho-1} b_{i} B_{i}$, where $b_{i} \in \mathbb{R}$ for every $i$. Since $D \cdot B_{i} \geqslant 0$ for every $i$ and $D \cdot \sigma^{*} E \geqslant 0$, $b_{i} \leqslant 0$ holds for every $i$. We assume that $D \neq 0$. Then we can take an irreducible curve $G$ on $\bar{S}$ such that $G \not \subset \operatorname{Supp} D$ and that $G \cap \operatorname{Supp} D \neq \emptyset$. This is because the smooth locus of $\bar{S}$ is a quasi-projective open subset of $\bar{S}$ and contains $\sigma^{*} E$ and $B_{i}$ for every $i$. Thus we get $D \cdot G<0$. This is a contradiction. This means that $D=0$. Hence we have $\overline{\operatorname{NE}}(\bar{S})=$ $N_{1}(\bar{S})=\mathbb{R}^{\rho}$.

If $C$ is an elliptic curve, then $\bar{S}$ is a complete nonprojective log canonical algebraic surface with $\overline{\mathrm{NE}}(\bar{S})=N_{1}(\bar{S})=\mathbb{R}^{\rho}$. By construction, we see that $K_{\bar{S}}=\sum_{i=1}^{\rho-1} B_{i}$. We can apply the minimal model program established in Theorem 1.5 to a complete nonprojective $\log$ canonical algebraic surface $\bar{S}$. Then every $B_{i}$ is contracted to a smooth point and we finally get a good minimal model $\widetilde{S}$, which is a surface with $K_{\widetilde{S}} \sim 0$ constructed in Example 12.4.

The following commutative diagram may help the reader understand the constructions in Examples 12.3-12.5:

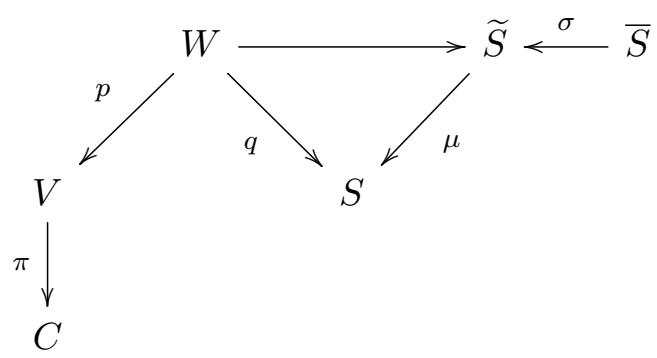

The reader can find various examples of complete nonprojective toric threefolds $X$ with $\operatorname{Pic}(X)=\{0\}, \overline{\mathrm{NE}}(X)=\mathbb{R}_{\geqslant 0}$, or $\overline{\mathrm{NE}}(X)=N_{1}(X)$ in [Fn1] and [FP]. 
Finally, we make a remark on complete nonprojective algebraic surfaces defined over an algebraically closed field $k$ with $k \neq \mathbb{C}$.

REMARK 12.6. We note that every complete algebraic surface defined over $\overline{\mathbb{F}}_{p}$ is always $\mathbb{Q}$-factorial (see [T, Theorem 4.5]). So it automatically becomes projective (see [Fn4, Lemma 2.2]). By the following lemma (see Lemma 12.7), the constructions in this section and Kollár's construction in [K1, Aside 3.46] can work for algebraic surfaces defined over an algebraically closed field $k$ such that $k \neq \overline{\mathbb{F}}_{p}$ for every prime number $p$ with some suitable modifications. We note that Theorem 3.11 holds true for algebraic surfaces defined over any algebraically closed field (see Remark 11.4). Thus, we can construct complete nonprojective algebraic surfaces over an algebraically closed field $k$ such that $k \neq \overline{\mathbb{F}}_{p}$ for every prime number $p$.

Lemma 12.7. Let $C$ be a smooth projective curve defined over an algebraically closed field $k$ whose genus $g(C)$ is positive. Let $P$ be an arbitrary closed point of $C$. Assume that $k \neq \overline{\mathbb{F}}_{p}$ for every prime number $p$. Then there exists $Q \in C$ such that $\mathcal{O}_{C}(Q-P)$ is a nontorsion element of $\operatorname{Pic}^{0}(C)$.

Proof. By $g(C) \geqslant 1$ and $k \neq \overline{\mathbb{F}}_{p}$, we can take a nontorsion element $\mathcal{L}$ of $\operatorname{Pic}^{0}(C)$ (see $[\mathrm{T}$, Fact 2.3]). We take a large positive integer $m$. Then $\mathcal{L} \otimes \mathcal{O}_{C}(m P)$ is very ample. We consider a general member $Q_{1}+\cdots+Q_{m}$ of $\left|\mathcal{L} \otimes \mathcal{O}_{C}(m P)\right|$. Then we have $\mathcal{L} \simeq \mathcal{O}_{C}\left(\left(Q_{1}-P\right)+\right.$ $\left.\cdots+\left(Q_{m}-P\right)\right)$. Therefore, there exists some $i_{0}$ such that $\mathcal{O}_{C}\left(Q_{i_{0}}-P\right)$ is a nontorsion element of $\operatorname{Pic}^{0}(C)$.

Acknowledgments. The author would like to thank Kenta Hashizume, Haidong Liu, and Hiromu Tanaka for very useful comments and for pointing out some mistakes. He would also like to thank Kento Fujita very much for useful discussions and advice, and for allowing him to use his ideas on complete nonprojective algebraic surfaces in Section 12. Finally, he thanks Seiko Hashimoto for her help and the referees for many comments.

\section{REFERENCES}

[BS] C. Bănică and O. Stănăşilă, Algebraic Methods in the Global Theory of Complex Spaces, John Wiley \& Sons, London-New York-Sydney, 1976, Translated from the Romanian, Editura Academiei, Bucharest.

[BHPV] W. P. Barth, K. Hulek, C. A. M. Peters and A Van de Ven, Compact Complex Surfaces, 2nd ed., Ergebnisse der Mathematik und ihrer Grenzgebiete. 3. Folge. A Series of Modern Surveys in Mathematics [Results in Mathematics and Related Areas. 3rd Series. A Series of Modern Surveys in Mathematics] 4, Springer-Verlag, Berlin, 2004.

[BCHM] C. Birkar, P. Cascini, C. D. Hacon and J. McKernan, Existence of minimal models for varieties of log general type, J. Amer. Math. Soc. 23(2) (2010), 405-468.

[CHP] F. Campana, A. Höring and T. Peternell, Abundance for Kähler threefolds, Ann. Sci. Éc. Norm. Supér. (4) 49 (2016), 971-1025.

[Fk] A. Fujiki, Kählerian normal complex surfaces, Tôhoku Math. J. (2) 35(1) (1983), 101-117.

[Fn1] O. Fujino, On the Kleiman-Mori cone, Proc. Japan Acad. Ser. A Math. Sci. 81(5) (2005), 80-84.

[Fn2] O. Fujino, Finite generation of the log canonical ring in dimension four, Kyoto J. Math. 50(4) (2010), 671-684.

[Fn3] O. Fujino, Fundamental theorems for the log minimal model program, Publ. Res. Inst. Math. Sci. $\mathbf{4 7}(3)$ (2011), 727-789.

[Fn4] O. Fujino, Minimal model theory for log surfaces, Publ. Res. Inst. Math. Sci. 48(2) (2012), 339-371.

[Fn5] O. Fujino, Some remarks on the minimal model program for log canonical pairs, J. Math. Sci. Univ. Tokyo 22(1) (2015), 149-192. 
[Fn6] O. Fujino, On log canonical rational singularities, Proc. Japan Acad. Ser. A Math. Sci. 92(1) (2016), 13-18.

[Fn7] O. Fujino, Foundations of the Minimal Model Program, MSJ Memoirs 35, Mathematical Society of Japan, Tokyo, 2017.

[FG] O. Fujino and Y. Gongyo, "On log canonical rings", in Higher Dimensional Algebraic Geometry-in honour of Professor Yujiro Kawamata's Sixtieth Birthday, Adv. Stud. Pure Math. 74, Math. Soc. Japan, Tokyo, 2017, 159-169.

[FL] O. Fujino and H. Liu, On the log canonical ring of projective plt pairs with the Kodaira dimension two, to appear in Ann. Inst. Fourier (Grenoble).

[FM] O. Fujino and S. Mori, A canonical bundle formula, J. Differential Geom. 56(1) (2000), 167-188.

[FP] O. Fujino and S. Payne, Smooth complete toric threefolds with no nontrivial nef line bundles, Proc. Japan Acad. Ser. A Math. Sci. 81(10) (2005), 174-179.

[FT] O. Fujino and H. Tanaka, On log surfaces, Proc. Japan Acad. Ser. A Math. Sci. 88(8) (2012), 109-114.

[Ft] T. Fujita, Fractionally logarithmic canonical rings of algebraic surfaces, J. Fac. Sci. Univ. Tokyo Sect. IA Math. 30(3) (1984), 685-696.

[Ha1] K. Hashizume, Finite generation of adjoint ring for log surfaces, J. Math. Sci. Univ. Tokyo 23(4) (2016), 741-761.

[Ha2] K. Hashizume, Minimal model theory for relatively trivial log canonical pairs, Ann. Inst. Fourier (Grenoble) 68(5) (2018), 2069-2107.

[HP1] A. Höring and T. Peternell, Mori fibre spaces for Kähler threefolds, J. Math. Sci. Univ. Tokyo 22(1) (2015), 219-246.

[HP2] A. Höring and T. Peternell, Minimal models for Kähler threefolds, Invent. Math. 203(1) (2016), 217-264.

[HP3] A. Höring and T. Peternell, "Bimeromorphic geometry of Kähler threefolds", in Algebraic Geometry: Salt Lake City 2015, Proc. Sympos. Pure Math. 97.1, Amer. Math. Soc., Providence, RI, 2018, 381-402.

[K1] J. Kollár, Lectures on resolution of singularities, Ann. Math. Stud. 166, Princeton University Press, Princeton, NJ, 2007.

[K2] J. Kollár, Singularities of the minimal model program, Cambridge Tracts in Mathematics 200, Cambridge University Press, Cambridge, 2013, With a collaboration of Sándor Kovács.

[KK] J. Kollár and S. Kovács, Birational geometry of log surfaces, preprint, 1994, available at https://w eb.math.princeton.edu/ kollar/.

[KM] J. Kollár and S. Mori, Birational Geometry of Algebraic Varieties, Cambridge Tracts in Mathematics 134, Cambridge University Press, Cambridge, 1998, With the collaboration of C. H. Clemens and A. Corti, Translated from the 1998 Japanese original.

[La] R. Lazarsfeld, Positivity in Algebraic Geometry. I. Classical Setting: Line Bundles and Linear Series, Ergebnisse der Mathematik und ihrer Grenzgebiete. 3. Folge. A Series of Modern Surveys in Mathematics [Results in Mathematics and Related Areas. 3rd Series. A Series of Modern Surveys in Mathematics] 48, Springer-Verlag, Berlin, 2004.

[Li] H. Liu, Some remarks on log surfaces, Proc. Japan Acad. Ser. A Math. Sci. 93(10) (2017), 115-119.

[Ma] K. Matsuki, Introduction to the Mori Program, Universitext, Springer-Verlag, New York, 2002.

[Mi] K. Miyamoto, The Sarkisov program on log surfaces, preprint, 2019, arXiv:1910.07025 [math.AG].

[Mo] S. Mori, "Classification of higher-dimensional varieties", in Algebraic Geometry, Bowdoin, 1985 (Brunswick, Maine, 1985), Proc. Sympos. Pure Math. 46, Part 1, Amer. Math. Soc., Providence, RI, 1987, 269-331.

[Mu1] D. Mumford, The topology of normal singularities of an algebraic surface and a criterion for simplicity, Inst. Hautes Études Sci. Publ. Math. 9 (1961), 5-22.

[Mu2] D. Mumford, "Enriques' classification of surfaces in char p. I", in Global Analysis (Papers in Honor of K. Kodaira), Univ. Tokyo Press, Tokyo, 1969, 325-339.

[Ng] M. Nagata, Existence theorems for nonprojective complete algebraic varieties, Illinois J. Math. 2 (1958), 490-498.

[Nk] N. Nakayama, Zariski-decomposition and abundance, MSJ Memoirs 14, Mathematical Society of Japan, Tokyo, 2004.

[P] P. Pascul Gainza, Ampleness criteria for algebraic spaces, Arch. Math. (Basel) 45(3) (1985), 270-274.

[S] F. Sakai, Weil divisors on normal surfaces, Duke Math. J. 51(4) (1984), 877-887. 
[T] H. Tanaka, Minimal models and abundance for positive characteristic log surfaces, Nagoya Math. J. 216 (2014), 1-70.

[U] K. Ueno, Classification Theory of Algebraic Varieties and Compact Complex Spaces, Lecture Notes in Mathematics 439, Springer-Verlag, Berlin-New York, 1975, Notes written in collaboration with P. Cherenack.

Department of Mathematics

Graduate School of Science

Osaka University

Toyonaka

Osaka 560-0043

Japan

fujino@math.sci.osaka-u.ac.jp 